\title{
Algorithms for Dense Graphs and Networks on the Random Access Computer ${ }^{1}$
}

\author{
J. Cheriyan ${ }^{2}$ and K. Mehlhorn ${ }^{3}$
}

\begin{abstract}
We improve upon the running time of several graph and network algorithms when applied to dense graphs. In particular, we show how to compute on a machine with word size $\lambda=\Omega(\log n)$ a maximal matching in an $n$-vertex bipartite graph in time $O\left(n^{2}+n^{2.5} / \lambda\right)=O\left(n^{2.5} / \log n\right)$, how to compute the transitive closure of a digraph with $n$ vertices and $m$ edges in time $O\left(n^{2}+n m / \lambda\right)$, how to solve the uncapacitated transportation problem with integer costs in the range $[0 . . C]$ and integer demands in the range $[-U . . U]$ in time $O\left(\left(n^{3}(\log \log / \log n)^{1 / 2}+n^{2} \log U\right) \log n C\right)$, and how to solve the assignment problem with integer costs in the range $[0 . . C]$ in time $O\left(n^{2.5} \log n C /(\log n / \log \log n)^{1 / 4}\right)$.

Assuming a suitably compressed input, we also show how to do depth-first and breadth-first search and how to compute strongly connected components and biconnected components in time $O\left(n \lambda+n^{2} / \lambda\right)$, and how to solve the single source shortest-path problem with integer costs in the range $[0 . . C]$ in time $O\left(n^{2}(\log C) / \log n\right)$. For the transitive closure algorithm we also report on the experiences with an implementation.
\end{abstract}

Key Words. Graph, Network, Algorithm, Dense graph, Dense network.

1. Introduction. We improve upon the running time of several graph and network algorithms when applied to dense graphs and networks by exploiting the parallelism on the word level available in random access computers [AV]. In particular, the bounds shown in Table 1 can be obtained for graphs and networks with $n$ vertices and $m$ edges on machines with word size $\lambda=\Omega(\log n)$. For several graph algorithms, we show that the previously best bounds can be improved by a factor $\lambda$ on dense graphs; e.g., a maximum matching in a bipartite graph can be computed in time $O\left(n^{2}+n^{2.5} / \lambda\right)=O\left(n^{2.5} / \log n\right)$. For problems on networks, e.g., the shortest-path problem, the assignment problem, and the transportation problem, assuming that all the numeric parameters of the network are integers, we obtain improvements by a fractional power of $\log n$ :

There is a simple common principle underlying all our improvements. This principle was introduced by Cheriyan et al. [CHM] in their $O\left(n^{3} / \log n\right)$ maximum-flow algorithm. Alt et al. [ABMP] showed later that the technique can also be applied to the bipartite matching problem. They obtained a running time of $O\left(n^{2.5} / \sqrt{\log n}\right)$. In this paper we further exploit the principle and show that it can be applied to a large number of graph and network problems.

\footnotetext{
${ }^{1}$ Most of this research was carried out while both authors worked at the Fachbereich Informatik, Universität des Saarlandes, Saarbrücken, Germany. The research was supported in part by ESPRIT Project No. 3075 ALCOM. The first author acknowledges support also from NSERC Grant No. OGPIN007.

${ }^{2}$ Department of Combinatorics and Optimization, University of Waterloo, Waterloo, Ontario N2L 3G1, Canada.

${ }^{3}$ Max-Planck-Institut für Informatik and Universität des Saarlandes, D-66123 Saarbrücken, Germany.
}

Received September 5, 1993; revised November 24, 1994. Communicated by R. Sedgewick. 
Table 1. Survey of results. ${ }^{1}$

\begin{tabular}{|c|c|c|c|}
\hline Problem & Running time & Input & Previous bound \\
\hline $\begin{array}{l}\text { Depth-first search } \\
\text { Breadth-first search } \\
\text { Strongly connected } \\
\text { components } \\
\text { Biconnected components }\end{array}$ & $O\left(n \lambda+n^{2} / \lambda\right)$ & c & $O\left(n^{2}\right)$ \\
\hline $\begin{array}{l}\text { Maximum matching } \\
\text { in bipartite graphs }\end{array}$ & $O\left(n^{2}+n^{2.5 / \lambda)}\right.$ & $\mathrm{s}$ & $O\left(n^{2.5}\right) \quad[\mathrm{HK}]$ \\
\hline Transitive closure & $O\left(n^{2}+n m / \lambda\right)$ & $\mathrm{s}$ & $O(n m)$ \\
\hline $\begin{array}{l}\text { Single source shortest } \\
\text { paths with edge } \\
\text { weights in }[0 . . C]\end{array}$ & $O\left(n^{2}(\log C / \log n)\right)$ & c & $O\left(n^{2}\right) \quad[\mathrm{D} 1]$ \\
\hline $\begin{array}{l}\text { Assignment problem } \\
\text { with edge } \\
\text { weights in }[0 . . C]\end{array}$ & $O\left(n^{2.5}(\log n C) \cdot \beta\right)$ & s & $O\left(n^{2.5} \log n C\right) \quad[\mathrm{GT}],[\mathrm{OA}]$ \\
\hline $\begin{array}{l}\text { Transportation } \\
\text { problem with } \\
\text { edge costs } \\
\text { in }[0 . . C] \text { and } \\
\text { demands in }[0 . . \mathrm{U}]\end{array}$ & $O\left(\left(n^{3} \gamma+n^{2} \log U\right) \log n C\right)$ & $\mathrm{s}$ & $\begin{array}{l}O\left(\left(n^{3}+n^{2} \log U\right) \log n C\right) \\
{[\mathrm{AGOT}]}\end{array}$ \\
\hline
\end{tabular}

The first column specifies the problem, the second column states the running time obtained in this paper ( $\beta$ denotes $(\log \log n / \log n)^{1 / 4}$ and $\gamma$ denotes $(\log \log n / \log n)^{1 / 2}$ ), the third column states whether the input is standard (s) or compressed (c), and the fourth column states the best previous bound. $\lambda$ denotes the word size of the machine.

The technique is most easily described in the case of depth-first search (DFS). DFS is a recursive procedure which explores a graph starting from a source $s$. Initially, all vertices are unlabeled and DFS $(s)$ is called. A call DFS $(v)$ labels $v$ and then scans the edges $(v, w)$ starting at $v$ until an unlabeled vertex $w$ is found. Then DFS $(w)$ is called. The crucial observation is that, although up to $n^{2}$ edges are examined by DFS, only $n-1$ of them lead to recursive calls. Suppose now that the adjacency matrix of the graph is available in "compressed form," i.e., $t, t \leq \lambda$, bits of the adjacency matrix are stored in a single computer word. Suppose also that we maintain the compressed bit-vector of unlabeled vertices. Then taking the componentwise AND of corresponding words of $v$ 's row of the adjacency matrix and the bit-vector of unlabeled vertices and testing the result for zero checks simultaneously for $t$ vertices whether one of them is unlabeled and reachable from $v$ by an edge. In this way the adjacency lists of all vertices can be scanned in $O\left(n^{2} / t\right)$ time. Only $n$ times will an edge leading to an unlabeled vertex be detected and a recursive call be required. This adds $O(n t)$ to the running time.

The details for DFS are given in Section 2.2. Breadth-first search is discussed in Section 2.3 , the computation of strongly connected and biconnected components in Section 2.4, the matching problem in Section 2.5, and the computation of transitive closures in Section 2.6. We mention that Feder and Motwani [FM] independently obtained an $O\left(n^{2.5} / \log n\right)$ bipartite matching algorithm. Their approach is quite different from ours. 
The algorithms for the computation of strongly and biconnected components given in Section 2.4 are alternatives to the algorithms in [T]. We find that the correctness proofs in Section 2.4 are more intuitive. For the transitive closure algorithm we also report on experiences with an implementation.

Section 3 is devoted to algorithms on networks; the shortest-path problem is discussed in Section 3.1, the transportation problem in Section 3.2, and the assignment problem in Section 3.3. For network algorithms, the compression technique requires the precomputation of tables and therefore typically the full word size cannot be exploited.

The machine model used in this paper is essentially the RAC (random access computer) of Angluin and Valiant [AV]. Let $\lambda$ be an integer. A $\lambda$-RAC consists of $M=2^{\lambda}$ registers, each of which can hold an integer in the range [0..M-1]. The instruction set of a $\lambda$-RAC consists of arithmetic operations (addition, subtraction, multiplication, and integer division (all modulo $M$ )) and boolean operations (AND, OR, EXCLUSIVE-OR, Negation). For the boolean operations an integer is interpreted as a bitstring of length $\lambda$; all boolean operations work bitwise, i.e., on all $\lambda$ bits in parallel. In contrast to Angluin and Valiant [AV], we do not postulate that the word size $\lambda$ is logarithmic in the size of the input. Rather, we treat word size and length of input as independent quantities and only require that the word size is at least logarithmic in the size of the input. Following Kirkpatrick and Reisch [KR], we call an algorithm conservative, if it uses only a word size which is logarithmic in the size of the input (although the actual word size of the machine in use may be larger).

\section{Graph Algorithms}

2.1. Basics. For an integer $t$ and $0-1$-valued vector $L[0 . n-1]$ the $t$-compression (or $t$-compressed version) $\diamond L$ of $L$ is a vector $\diamond L[0 . .[n / t\rceil-1]$ such that, for $0 \leq k<\lceil n / t\rceil$,

$$
\diamond L[k]=\sum_{0 \leq l<t} L[k \cdot t+l] 2^{t-l},
$$

where $L[v]=0$ for $v \geq n$ is assumed for simplicity. The entries of a $t$-compression take values in $T=\left[0 . .2^{t}-1\right]$.

For integers $x \in T$ and $l, 0 \leq l<t$, we use $(x)_{l}$ to denote the $l$ th bit of $x$, i.e., $x=\sum_{0 \leq l<t}(x)_{l} \cdot 2^{l}$ and $(x)_{l} \in\{0,1\}$ for $0 \leq l<t$. For $0 \leq l<t$ let $E_{l}$ denote the integer $2^{l}$.

For $x \in T, x \neq 0,\lfloor\log x\rfloor$ is the index of the highest numbered nonzero bit in $x$. In our graph algorithms we frequently have to compute $\lfloor\log x\rfloor$. We assume that $\lfloor\log x\rfloor$ is not available as a machine instruction. The simplest algorithm is linear search.

$$
l \leftarrow t-1 \text {; while }\left(x \text { AND } E_{l}\right)=0 \text { do } l \leftarrow l-1 \text { od }
$$

It takes time $O(t)$ and needs no precomputation. A faster method is binary search. It takes $O(\log t)$ time and requires the precomputation of $O(t)$ masks. Finally, Freedman and Willard [FW] have recently found a method which works in time $O(1)$ with $O(\lambda)$ precomputation. In the algorithms below we always state the time bounds in terms of linear search. 
For a graph $G=(V, E)$ with $n$ nodes we identify the vertices with the integers $0,1, \ldots, n-1$ and we also use $E$ to denote the adjacency matrix of $G$, i.e., $E[v, w]=1$ iff $(v, w) \in E$, and $E[v, w]=0$, otherwise. The $t$-compressed adjacency matrix $\diamond E$ is a matrix $\diamond E[0 . . n-1,0 . .\lceil n / t\rceil-1]$ such that the $v$ th row of $\diamond E$ is the $t$-compression of the $v$ th row of $E, 0 \leq v<n$.

2.2. Depth-First Search. Depth-first search (DFS) is a useful method for the systematic exploration of a graph. DFS visits the nodes of a graph in depth-first order, i.e., DFS always follows an unexplored edge (if any) out of the most recently reached vertex. Program 1 specifies DFS as a recursive procedure $d f($ node $v$ ). This program also computes two node labelings $d f$ snum and compnum and a list of tree edges. The labeling $d f$ snum numbers the nodes by the time of the call of $d f s$, compnum numbers the nodes by the time of the completion of the call of $d f s$, and tree contains the set of edges whose exploration leads to recursive calls. DFS runs in time and space $O\left(n^{2}\right)$ on an $n$-vertex graph.

We now describe the compression technique. Let $\diamond E$ be the $t$-compressed adjacency matrix. We also store the bit-vector reached in its $t$-compressed form $\diamond$ reached and represent a node $v$ by the pair $(i, j)$ with $i=\lfloor v / t\rfloor$ and $j=v \bmod t$. The crucial observation is now that, for $0 \leq h \leq\lceil n / t\rceil-1, \diamond E[v, k] \wedge \neg \diamond$ reached $[k] \neq 0$ iff some edge in $\{(v, w) ; k t \leq w<(k+1) t\}$ leads to an unreached node, i.e., one operation checks $t$ edges. The details are given in Program 2.

LEMMA 1. Given the $t$-compressed adjacency matrix $\diamond E$ of an $n$-vertex graph, $t \leq \lambda$, depth-first search runs in time $O\left(n^{2} / t+n t\right)$ and space $O\left(n^{2} / t\right)$ on a $\lambda-R A C$.

ProOF. The time spent outside line $5 \mathrm{c}$ is clearly $O\left(n^{2} / t\right)$. Also, a single execution of line $5 \mathrm{c}$ takes time $O(t)$ and there are at most $n-1$ executions of it since there are

(1) procedure $d f s$ (node $v$ )

(2) begin reached $[v] \leftarrow 1$;

(3) $\quad d f$ snum $[v] \leftarrow d f s_{-}$count $1 \leftarrow d f s_{-}$count $1+1$;

(4) for $w \in V$

(5) do if $E[v, w]$ and $\neg \operatorname{reached}[w]$

(6) then $T$.append $((v, w))$;

(7) $\quad d f_{s}(w)$

(8) fi

(9) od;

(10) compnum $[v] \leftarrow d s_{-}$count $2+1$

(11) end

(12) $T \leftarrow$ empty list of edges

(13) for $v \in V$ do reached $[v] \leftarrow 0$ od;

(14) $d f s_{-}$count $1 \leftarrow d f s_{-}$count $2 \leftarrow-1$;

(15) for $v \in V$

(16) do if $\neg$ reached $[v]$ then $d f s(v)$ fi od

Program 1. Depth-first search. 


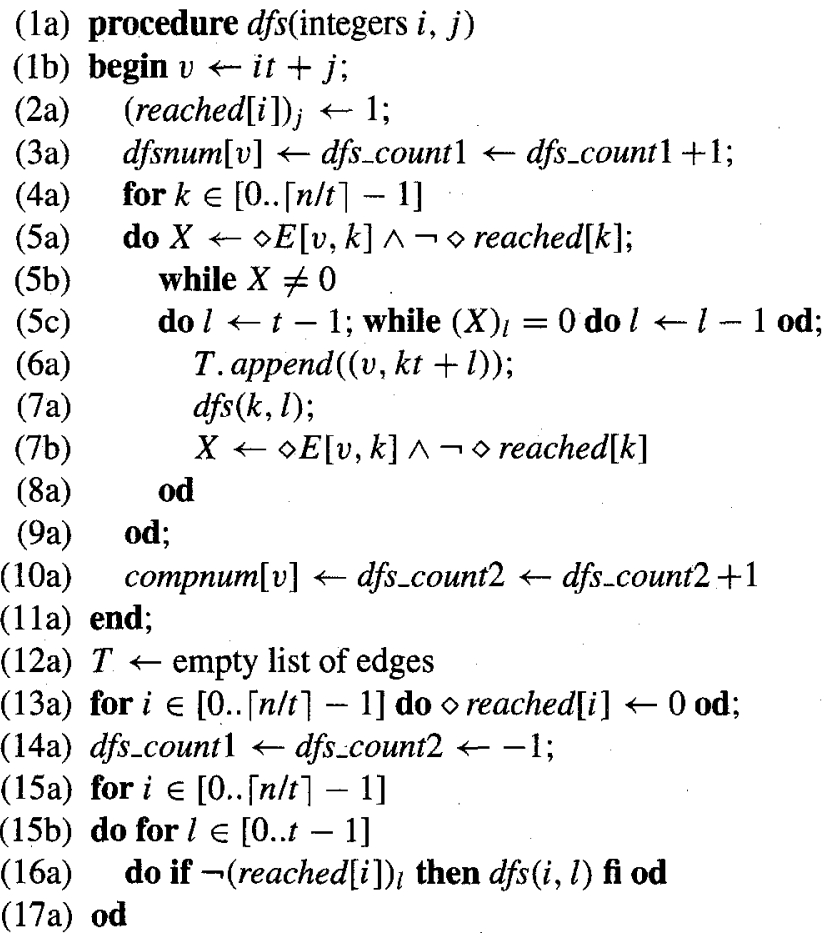

Program 2. Depth-first search with compressed adjacency matrix.

at most $n-1$ calls to $d f s$ in line $7 \mathrm{a}$. This proves the time bound. The space bound is obvious.

REMARK. Line $5 \mathrm{c}$ computes $\lfloor\log X\rfloor$ by linear search in time $O(t)$. In view of Section 2.1, we may also use binary search or the constant time method of Fredman and Willard provided that we add $O(t)$ and $O(\lambda)$ preprocessing time, respectively. This gives a running time of $O\left(n^{2} / t+n \log t+t\right)$ and $O\left(n^{2} / t+\lambda\right)$, respectively. We prefer to state our time bounds in terms of linear search because it uses the weakest machine model.

DFS can be used to partition the edges of a graph into tree, forward, back, and cross edges. The tree edges have already been collected in the list $L$ in Program 1. We now show how to construct the submatrices of $\diamond E$ corresponding to the three other classes of edges. All three classes can be characterized in terms of the two labelings $d f$ snum and compnum, see [T] and Section IV.5 of [M], e.g., an edge $(v, w)$ is a cross edge if $d f_{\text {snum }}[v]>d f_{\text {snum }}[w]$ and compnum $[v]>\operatorname{compnum}[w]$. We can therefore extract the submatrix of cross edges by deleting all edges which violate one of the two defining conditions. The following simple strategy deletes for example all edges $(v, w)$ with $d f$ snum $[v] \leq d f s n u m[w]$. We step through the vertices in increasing order of $\mathrm{dfs}$ number and maintain the $t$-compresssion $\diamond$ smaller of a bit-vector smaller with smaller $[w]=1$ iff $d f_{s n u m}[w]<d f$ snum $[v]$. The AND of $\diamond E[v, *]$ and $\diamond$ smaller then deletes all edges 
$(v, w)$ with $d f \operatorname{snum}[v] \leq d f \operatorname{snum}[w]$. The program follows:

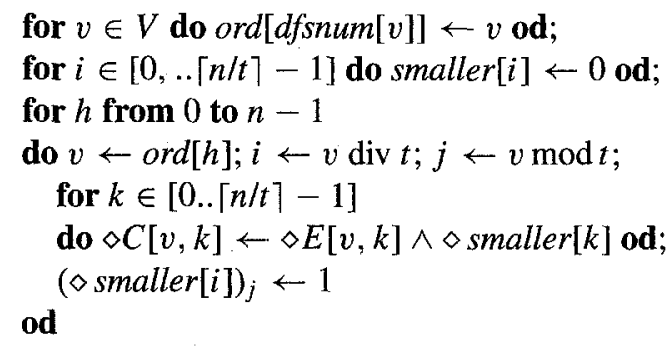

LEMMA 2. Under the hypothesis of Lemma 1, the $t$-compressed adjacency matrices for the forward, back, and cross edges can be computed in time $O\left(n^{2} / t+n t\right)$ on a $\lambda-R A C$.

2.3. Breadth-First Search. Breadth-first search (BFS) computes the shortest distances of all nodes from a given set $S$ of nodes, i.e., a node labeling $d$ with

$$
\begin{gathered}
d(v)=\min \left\{k ; \exists v_{0}, v_{1}, \ldots, v_{k} \text { such that } v_{0} \in S, v_{k} \in v,\right. \\
\text { and } \left.\left(v_{i}, v_{i+1}\right) \in E \text { for } 0 \leq i<k\right\},
\end{gathered}
$$

see Program 3. BFS takes time $O\left(n^{2}\right)$ on an $n$-vertex graph. With the methods of Section 2.2 (the details are left to the reader) this can be improved to $O\left(n^{2} / t+n t\right)$ time on a $\lambda$-RAC, provided that $t \leq \lambda$ and the $t$-compressed adjacency matrix is given.

The layered subgraph of a graph $G$ consists of all edges $(v, w)$ with $d[w]=d[v]+1$. It is needed in several applications of BFS, e.g., to matching or flow problems; see

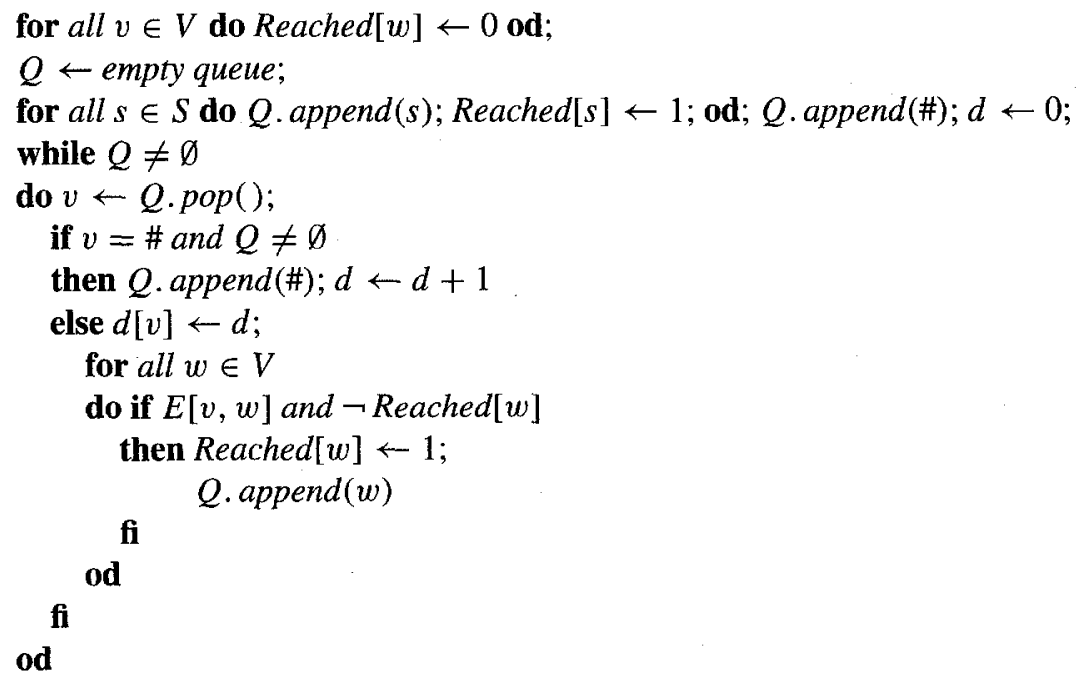

Program 3. Breadth-first search; $Q$. append $(x)$ appends $x$ to the rear of $Q, Q \cdot p o p()$ deletes the first element of $Q$ and returns it. 
Section 2.5. We now discuss how to construct the compressed adjacency matrix of the layered subgraph. For $k, 0 \leq k<n$, let $\diamond L_{k}$ be the $t$-compression of the bit-vector $L_{k}$ where $L_{k}[v]=1$ iff $d[v]=k$ for all $v \in V$. These vectors can be computed in time $O\left(n^{2} / t+n\right)$; namely $O\left(n^{2} / t\right)$ time to initialize them to zero and $O(1)$ time for each vertex. Next observe that the $v$ th row of the $t$-compressed adjacency matrix $\diamond D$ of the layered subgraph is given by the AND of $\diamond E[v, *]$ and $L_{d[v]+1}$. We summarize in:

LEMMA 3. Given the $t$-compressed adjacency matrix of an $n$-vertex graph, $t \leq \lambda$, and a subset $S$ of the vertices, BFS and the construction of the layered subgraph of $G$ take time $O\left(n^{2} / t+n t\right)$ on $a \lambda-R A C$.

2.4. Strongly Connected and Biconnected Components. A digraph $G=(V, E)$ is strongly connected if for any two vertices $v, w \in V$. there is a path from $v$ to $w$. A strongly connected component (scc) is a maximal strongly connected subgraph. An undirected graph $G=(V, E)$ is biconnected if for any two edges $e$ and $e^{\prime}$ there is a simple cycle containing $e$ and $e^{\prime}$. A biconnected component (bcc) is a maximal biconnected subgraph.

Tarjan [T] has given linear-time algorithms for the computation of scc's and bcc's. Both algorithms are based on the computation of so-called lowpoints. Since lowpoint values can change $\Omega(m)$ times, his algorithms do not seem amenable to the techniques described in the previous sections. Another linear-time algorithm for the computation of scc's was given by Sharir [Sh]. It uses DFS on $G$ and $G^{\text {rev, }}$, the graph obtained from $G$ by reversal of all edges. Section 2.2 implies that Sharir's algorithm runs in time $O\left(n^{2} / t+n t\right)$ provided that the $t$-compressed adjancency matrix of $G$ and $G^{r e v}$ are given. Unfortunately, Sharir's algorithm cannot be used to compute bcc's of undirected graphs.

In this section we describe an $O(m)$ algorithm for the computation of scc's which is as fast as Tarjan's algorithm (Sharir's is slower by about a factor of two), is simple (maybe even simpler than Tarjan's algorithm), can be modified to compute bcc's, and can be made to run in time $O\left(n^{2} / t+n t\right)$ given the $t$-compressed adjacency matrix. Our algorithm is similar to an algorithm described by Dijkstra [D2] which has however running time $\Omega\left(n^{2}\right)$.

Our algorithm is based on DFS and constructs the scc's of $G$ incrementally. Let $G_{c u r}$ be the subgraph of $G$ consisting of all vertices reached by DFS and all edges explored by DFS. An scc of $G_{c u r}$ is called completed if the call $d f s(v)$ is completed for all vertices $v$ of the component, and uncompleted, otherwise. Let unfinished $=\left(v_{1}, v_{2}, \ldots, v_{s}\right)$ be the sequence of vertices of $G_{c u r}$ in uncompleted components of $G_{c u r}$ ordered according to increasing DFS number. For each scc $C$ call the node with the smallest DFS number the root of $C$, and let roots $=\left(v_{i_{1}}, v_{i_{2}}, \ldots, v_{i_{k}}\right)$ with $1=i_{1}<i_{2}<\cdots<i_{k}$ be the subsequence of unfinished consisting of the roots of the uncompleted components. We maintain the following three invariants.

- I1: There are no edges $(x, y)$ of $G_{c u r}$ with $x$ belonging to a completed component and $y$ belonging to an uncompleted component.

- I2: The nodes in roots lie on a single tree path, i.e., $v_{i_{e}} \underset{r^{*}}{\longrightarrow} v_{i_{l}+1}$ for $1 \leq l<k$, and we are currently exploring edges out of $v_{p}$ where $p \geq i_{k}$. 

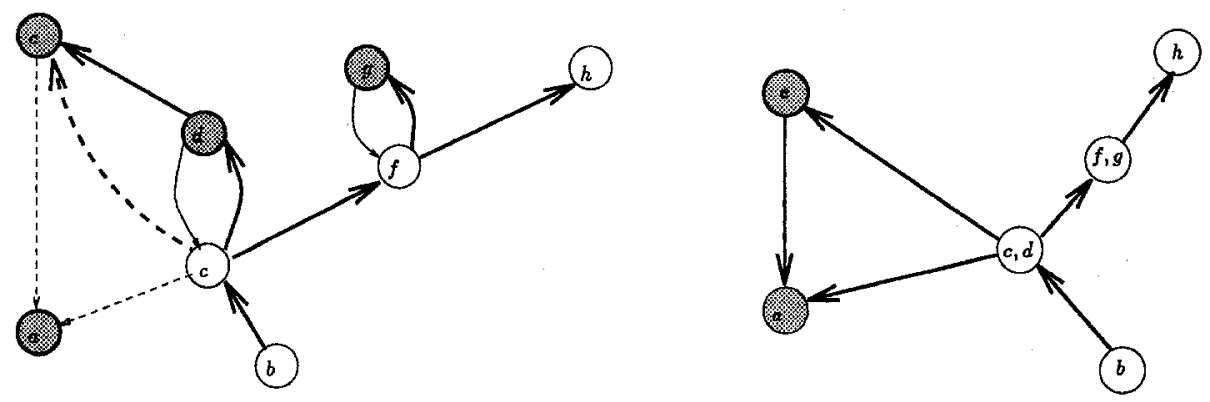

Fig. 1. A graph $G_{c u r}$; tree (back, cross, forward) edges are shown thick solid (thin solid, thin dashed, thick dashed). Nodes for which the call of $d f s$ is completed are shaded. The shrunken graph is also shown. Completed components are shaded.

- I3: The nodes in the uncompleted scc with root $v_{i_{l}}$ are the nodes $v_{i_{l}}, v_{i_{l}+1}, \ldots, v_{i_{l+1}-1}$ (with the convention $i_{k+1}=s+1$ ). Moreover, all these nodes are tree descendants of the root $v_{i_{l}}$.

EXAMPLE. Assume that $G_{c u r}$ is as shown in Figure 1. We assume that DFS reaches the vertices in the order $a, b, c, d, e, f, g, h$, that the calls $d f s(a), d f s(e), d f s(d), d f s(g)$ are completed, and that we are currently exploring edges out of vertex $h$. We have unfinished $=(b, c, d, f, g, h)$ and roots $=(b, c, f, h)$.

We now consider the exploration of edges and the completion of calls. If $(v, w)$ is the edge to be explored, let $G_{c u r}^{\prime}=\left(V_{c u r} \cup\{w\}, E_{c u r} \cup\{(v, w)\}\right)$ be the new graph spanned by the explored edges. Of course, $w \in V_{c u r}$ if $(v, w)$ is not a tree edge.

EXPLORATION OF A TREE EDGE $(v, w)$. In $G_{c u r}^{\prime}$ the node $w$ is a scc by itself and, of course, an uncompleted one; all other scc's stay the same. We can reflect this change by adding the node $w$ at the end of sequences unfinished and roots. Note that this preserves all our invariants. I3 is preserved since $w$ is a scc by itself. I1 is preserved since the node $v$ belongs to an uncompleted component according to $\mathrm{I} 3 ; \mathrm{I} 2$ is preserved since $v$ is a tree descendant of the last element of sequence roots according to $\mathrm{I} 2, \mathrm{I} 3$, and the fact that $(v, w)$ is a tree edge. Also, the sequences unfinished and roots are still ordered by DFS number.

In Program 4 lines 3 and 4 implement the actions described above. The sequence roots and unfinished are realized as pushdown stores; in addition, unfinished is also represented as a boolean array in_unfinished.

EXPLORATION OF A NONTREE EDGE $(v, w)$. We have to distinguish two cases: either $w$ belongs to a completed component or it does not. The case distinction is made in line 8 of Program 4. 
(1) procedure $d f s(v:$ node);

(2) count $1 \leftarrow$ count $1+1$; dfsnum $[v] \leftarrow$ count 1 ; reached $[v] \leftarrow$ true;

(3) push $v$ onto unfinished; in_unfinished $[v] \leftarrow$ true;

(4) push $v$ onto roots;

(5) for all $w$ with $(v, w) \in E$

(6) do if $\neg$ reached $[w]$

(7) then $d f s(w)$

(8) else if in_unfinished $[w]$

(9) then co we now merge components oc

(10) while $d f_{\text {snum }}[$ top (roots) $]>d f_{\text {snum }[w]}$

(11) do pop (roots) od

(12) $\quad$ i

(13) $\mathbf{f i}$

(14) od;

(15) if $v=t o p($ roots $)$

(16) then repeat $w \leftarrow$ pop (unfinished); in_unfinished $[w] \leftarrow$ false;

(18) until $v=w$;

(19) pop(roots)

(20) $\mathbf{f i}$

(21) end;

(22) begin co main program oc

(23) unfinished $\leftarrow$ roots $\leftarrow$ empty stack;

(24) count $1 \leftarrow 0$;

(25) for all $v \in V$ do in_unfinished $[v] \leftarrow$ false; reached $[v] \leftarrow$ false; od;

(26) for all $v \in V$ do if $\neg$ reached $[v]$ then $d f s(v)$ fi od

(27) end.

Program 4. An sec algorithm.

Case 1: $w$ belongs to a completed component. In this case no path exists from $w$ to $v$, since $v$ belongs to an uncompleted component of $G_{c u r}$ according to $\mathbf{L}$ and no edge exists from a node in a completed component to a node in an uncompleted component according to I1. Thus $G_{c u r}^{\prime}$ and $G_{c u r}$ have the same scc's and no action is required. The three invariants are clearly preserved.

Case 2: $w$ belongs to an uncompleted component. Let unfinished $=\left(v_{1}, v_{2}, \ldots, v_{s}\right)$ and let roots $=\left(v_{i_{1}}, v_{i_{2}}, \ldots, v_{i_{k}}\right)$, where $1=i_{1}<i_{2}<\ldots<i_{k}$. Let $v=v_{p}$, where $p \geq i_{k}$ according to 12 , and $w=v_{q}$ where $i_{l} \leq q<i_{l+1}$, i.e., $v_{i,}$ is the root of the scc containing $w$. Then the scc's of $G_{c u r}^{\prime}$ can be obtained by merging the scc's of $G_{c u r}$ with roots $v_{i_{l}}, v_{i_{i+1}}, \ldots, v_{i_{k}}$ into a single scc with root $v_{i_{l}}$ and leaving all other scc's unchanged. This can be seen as follows. Note first that completed scc's remain the same according to I1. Next consider any node $z$ in an uncompleted component, i.e., $z=v_{r}$ for some $r$. 
If $r \geq i_{l}$, say $i_{h} \leq r<i_{h+1}$ with $l \leq h \leq k$, then

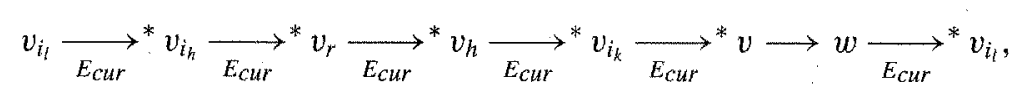

where the existence of the first, the fourth, and the fifth path follows from I2 and I 3 , the existence of the second and third path follows from the fact that $v_{i_{k}}$ and $v_{r}$ belong to the same scc, and the existence of the seventh path follows from the fact that $w$ and $v_{i_{i}}$ belong to the same sce. Thus $v_{r}$ and $v_{i_{i}}$ belong to the same scc of $G_{c u r}^{\prime}$ if $r \geq i_{l}$.

If $r<i_{h}$, say $i_{h} \leq r<i_{h+1}$ with $h<l$, then $v_{r} \underset{E_{\text {cur }}}{\longrightarrow} v_{i_{h}} \underset{E_{\text {Cur }}}{\longrightarrow} v_{i_{l}} \underset{E_{\text {cur }}}{\longrightarrow} w$, since $v_{r}$ and $v_{i_{h}}\left(v_{i_{i}}\right.$ and $w$ respectively) belong to the same scc and $v_{i_{h}}{\overrightarrow{E_{C u r}}}^{*} v_{i_{t}}$ according to 12. Since $h<l$ no path exists from $v_{i_{l}}$ to $v_{r}$ in $G_{c u r}$. If there were such a path in $G_{c u r}^{\prime}$, then it would have to use the edge $(v, w)$ and hence there would have to be a path from $w$ to $v_{r}$ in $G_{c u r}$. Thus $w$ and $v_{r}$ would belong to the same scc of $G_{c u r}$, a contradiction. This shows that uncompleted scc's with roots $v_{i_{h}}, h<l$, remain unchanged,

We have now shown that the scc's of $G_{c u r}^{\prime}$ can be obtained from the scc's of $G_{c u r}$ by merging the scc's with roots $v_{i_{i}}, \ldots, v_{i_{h}}$ into a single scc. The newly formed scc has root $v_{i_{i}}$ and hence the merge can be achieved by simply deleting the roots $v_{i_{+1}}, \ldots, v_{i_{k}}$ from roots. Next note that $i_{l} \leq q<i_{i+1}<\cdots<i_{k}$, where $w=v_{q}$ and hence $d$ fsnum $\left[v_{i_{1}}\right] \leq d$ fsnum $[w]<d f$ snum $\left[v_{i_{i+1}}\right]<\cdots<d$ fsnum $\left[v_{k}\right]$ since unfinished and roots are ordered according to DFS number. This shows that the merge can be achieved by popping all roots from roots which have a DFS number larger than $w$. That is exactly what lines 10 and 11 of Program 4 do. The three invariants are preserved by the arguments above. This finishes the description of how edges are explored. We now turn to the completion of calls.

COMPLETION OF A CALL $d f s(v)$. According to 12 the node $v$ is a tree descendant of the last vertex of roots, $i_{k}=t o p($ roots $)$. If it is a proper tree descendant, i.e., $v \neq$ top (roots), then the completion of $d f s(v)$ does not complete an scc. We return to $d f s(w)$ where $w$ is the parent of $v$. Clearly, $w$ is still a tree descendant of top(roots) and also $w{\overrightarrow{E_{\text {cur }}}}^{*} v{\overrightarrow{E_{\text {cur }}}}^{*} \operatorname{top}($ roots) belong to the same sec. This shows that $\mathrm{I} 2$ and $\mathrm{I} 3$ are preserved; I1 is also preserved since we do not complete a component.

If $v=t o p$ (roots), then we complete a component. According to 13 this component consists of exactly those nodes in unfinished which do not precede top (roots) and hence these nodes are easily enumerated as shown in lines 16-18 of Program 4. Of course, top (roots) ceases to be a root of an uncompleted scc and hence has to be deleted from roots; line 19. We still need to prove that the invariants are preserved. For I1 this follows from the fact that all edges leaving the just completed scc must terminate in previously completed scc's, since the uncompleted scc's form a path according to 12 . The invariants 12 and $\mathrm{I} 3$ are also maintained by a similar argument as in the case $v \neq t o p($ roots).

We have now proved the correctness of Program 4 and summarize in:

THEOREM 1. Program 4 computes the strongly connected components of a digraph in time $O(n+m)$. 
PROOF. Having already proved correctness, we still have to prove the time bound. The time bound follows directly from the linear time bound for DFS and the fact that every node is pushed onto and hence popped from unfinished and roots exactly once. This implies that the time spent in lines 11 and 16 is $O(n)$. The time spent in all other lines is $O(n+m)$.

We next discuss a more efficient implementation of this algorithm for dense graphs. It is based on the observation that at most $2(n-1)$ edges lead to a recursive call or to a merge of existing components. Our goal is therefore to identify these edges quickly. For each root $r$ of an uncompleted component let $B_{r}$ be a bit-vector such that $B_{r}[v]=1$ iff $v$ belongs to the uncompleted component with root $v$. The exploration of an edge $(v, w)$ leads to a recursive call $d f_{s}(w)$ if reached $[w]=0$ and it causes some components to be merged if in $_{-}$unfinished $[w]=1$ and $B_{\text {top(roots })}[w]=0$, i.e., if $w$ lies in an uncompleted component which is not the component of top(roots). If all bit-vectors are stored in $t$-compressed form, then this condition can be tested in time $O(1)$ for a block of $t$ edges and hence the time spent on scanning adjacency lists is $O\left(n^{2} / t+n t\right)$. When a new vertex $w$ is reached and $d f s(w)$ is called we create a new $t$-compressed vector $\diamond B_{w}$ and initialize it such that $B_{w}[x]=1$ iff $w=x$. This takes time $O(n / t)$ for each call and hence $O\left(n^{2} / t\right)$ in total. When two components are merged, we also need to update the $B$-vectors, i.e., line 11 is changed into

$$
\begin{aligned}
& B \leftarrow B_{t o p(r o o t s)} \\
& \text { pop roots; } \\
& B_{\text {top(roots })} \leftarrow B_{t o p(\text { roots })} \vee B
\end{aligned}
$$

This takes time $O(n / t)$ for each merge step and hence $O\left(n^{2} / t\right)$ in total. We summarize in:

THEOREM 2. Given the $t$-compressed adjacency matrix of an $n$-vertex graph, $t \leq \lambda$, the strongly connected components of $G$ can be computed in time $O\left(n^{2} / t+n t\right)$ on $a$ $\lambda-R A C$.

We next turn to the computation of biconnected components of undirected graphs which we assume to be given by their (symmetric) adjacency matrix. For a bcc $C$ we call the vertex with the second smallest DFS number the center of $C$, and for each vertex $w$ let parent $[w]$ be the parent of $w$ in the DFS tree. A bcc $C$ is called completed if the call $d f s(v)$ where $v$ is the center of $C$ is completed. As before, let unfinished denote the sequence of vertices belonging to uncompleted bcc's of $G_{c u r}$ in increasing order of DFS number. Note that a vertex can belong to several bcc's; it stays in unfinished until all of them are completed. Finally, centers is the subsequence of centers in unfinished. The invariants are now:

- I1: For all edges $(x, y)$ of $G_{c u r}, x$ and $y$ belong to the same bcc of $G_{c u r}$.

Let unfinished $=\left(v_{1}, v_{2}, \ldots, v_{k}\right)$ and centers $=\left(v_{i_{1}}, v_{i_{2}}, \ldots, v_{i_{s}}\right)$, where $i_{1}<i_{2}<$ $\cdots<i_{k}$. 
- 12: The vertices in centers lie on a single tree path and we are currently exploring edges out of $v_{p}$ where $p \geq i_{k}$.

- 13: The vertices in the uncompleted bcc with center $v_{i_{l}}$ are the vertices $v_{i_{l}}, v_{i_{l}+1}, \ldots$, $v_{i_{t}+1}$ (with the convention $i_{k}=s$ ) together with the vertex father $\left[v_{i_{l}}\right]$. All but the vertex father $\left[v_{i_{l}}\right]$ are tree descendants of $v_{i_{l}}$.

In the program line 4 is changed into

(4a) push $v$ onto centers,

lines 10 and 11 into

(10a) while $d f$ snum[father[top(centers) $]]>d f$ snum $[w]$

(11a) do pop (centers) od

and lines 15-20 into

(15a) if $v=\operatorname{top}($ centers)

(16a) then repeat $w \leftarrow$ pop (unfinished); in_ unfinished $[w] \leftarrow$ false

(17a) until $w=v$;

(18a) pop(centers);

(19a) ( ${ }^{*}$ father $[v]$ and the vertices just popped from the bcc with center $\left.c^{*}\right)$

(20a) fi.

THEOREM 3. The program above computes the biconnected components of an undirected graph in time $O(n+m)$. Given the $t$-compressed adjacency matrix, $t \leq \lambda$, it can be made to run in time $O\left(n^{2} / t+n t\right)$ on a $\lambda-R A C$.

Proof. Analogous to the proofs of Theorems 1 and 2.

The strongly connected components algorithm (without compression) described above and Tarjan's algorithm using lowpoints are part of the LEDA platform of combinatorial and geometric computing [MN], [N]. The running times of both algorithms are about the same.

2.5. Maximum Bipartite Matching. The maximum bipartite matching problem (MPM problem) is to find a maximum cardinality matching in a bipartite graph. An undirected graph $G=(V, E)$ is bipartite if there is a partition of the vertex set $V$ into disjoint sets $A$ and $B$ such that every edge $e \in E$ has exactly one endpoint in each of the two sets. A matching $M$ is a subset of $E$ such that every vertex is incident to at most one edge in $M$.

Hopcroft and Karp [HK] have shown how to solve the MPM problem in time $O\left(n^{1 / 2} \cdot m\right)$. We give an implementation of their algorithm which runs in time $O\left(n^{1 / 2}\left(n^{2} / \lambda+n \lambda\right)\right)$ on a $\lambda$-RAC. Thus the MPM problem can be solved in time $O\left(n^{2.5} / \log n\right)$ by a conservative algorithm. For dense graphs, this improves upon [HK] and [ABMP].

The algorithm of Hopcroft and Karp works in $O(\sqrt{n})$ phases. In each phase, which takes $O(m)$ time, a maximal set (with respect to set inclusion) of shortest augmenting paths is determined by BFS and subsequent DFS.

An alternating path is a path in $G$ which alternately uses edges in $M$ and $E-M$. 
An augmenting path with respect to a matching $M$ is an alternating path connecting two free vertices in $V$, i.e., vertices which are not incident to an edge in $M$. Interchanging the matching and nonmatching edges of an augmenting path increases the cardinality of the matching by one.

We can now describe a phase of their algorithm in more detail. Let $M$ be the matching at the beginning of the phase. Let $G_{M}=\left(V, E_{M}\right)$ be a directed graph with edge set $E_{M}=\{(v, w) ;\{v, w\} \in E \backslash M, v \in A, w \in B\} \cup\{(w, v) ;\{v, w\} \in M, v \in A, w \in B\}$, i.e., the edges in $M$ are directed from $B$ to $A$ and the edges outside $M$ are directed from $A$ to $B$. Clearly, the paths from free vertices in $A$ to free vertices in $B$ are in one-to-one correspondence to the augmenting paths with respect to $M$. In each phase a BFS of $G_{M}$ starting from the free vertices in $A$ is carried out first. Let $d$ be the minimal distance label of a free vertex in $B$, let $G_{L}$ consist of the layers 0 through $d$ of the layered subgraph of $G_{M}$, and let $D$ be the adjacency matrix of $G_{L}$. Clearly, all shortest augmenting paths with respect to $M$ can be found in $G_{L}$. A maximal set of vertex-disjoint augmenting paths can be determined by a variant of DFS, see Program 5. It maintains a set $L$ of vertex-disjoint augmenting paths (initially empty) and a set of reached vertices. A call search_path $(v)$, where $v \in A$ is free, constructs an augmenting path from $v$ to a free node in $B$ (if any) and adds it to $L$. Also, all nodes visited by the search are added to the set of reached vertices. Having determined a maximal set $L$ of vertex-disjoint augmenting paths, the matching $M$ is updated by reversing the direction of all edges of all paths in $L$. Program 5 describes the search for augmenting paths, and the procedure search_path, used by the search, is described in Program 6.

We now discuss how to implement a phase in time $O\left(n^{2} / \lambda+n \lambda\right)$ on a $\lambda$-RAC. We assume inductively that the $\lambda$-compressed adjacency matrix of $G_{M}$ is available at the beginning of a phase. (For $M=\emptyset$, it takes time $O\left(n^{2}\right)$ to establish this assumption). We first construct the $\lambda$-compressed adjacency matrix $\diamond D$ of $G_{L}$ using BFS as described in Section 2.2 in time $O\left(n^{2} / \lambda+n \lambda\right)$, and then search for augmenting paths as described above. Also, we maintain the array reached as a compressed array. Since search_path is called at most once for each vertex and since the total length of the augmenting paths found in one phase is at most $n$, the time spent for the search is $O(n)$ except for the three lines marked by ( $\diamond)$ in Program 6. Replacing them by

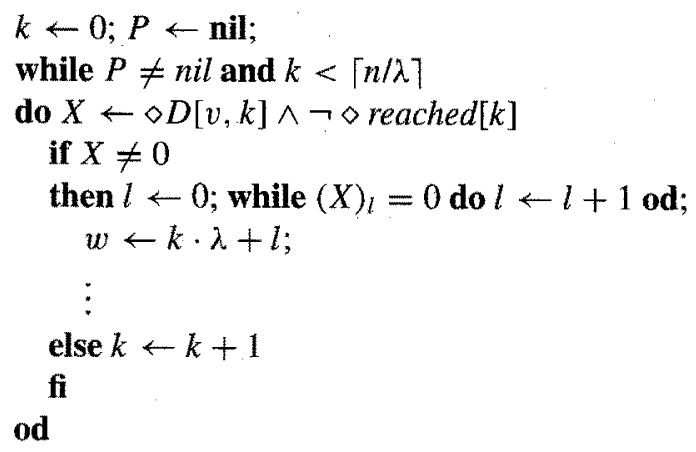

brings the cost of these lines down to $O\left(n^{2} / \lambda+n \lambda\right)$. Finally, reversing the direction of all edges of all paths in $L$ takes time $O(n)$. 
$L \leftarrow$ empty set of paths;

for all $v \in V$ do reached $[v] \leftarrow 0$ od;

for all $v \in A, v$ free

do $\left({ }^{*} L\right.$ is a set of vertex-disjoint augmenting paths; reached $[v]=1$ implies that either $v$ lies on a path in $L$ or there is no path from $v$ to a free vertex in $B$ disjoint from the paths in $L^{*}$ )

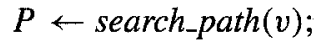

if $P \neq$ nil then L.append $(P) \mathbf{f i}$

od

Program 5. Searching for augmenting paths.

We summarize in:

THEOREM 4.

(a) On a $\lambda$-RAC a maximum matching in an $n$-vertex bipartite graph can be computed in time $O\left(n^{1 / 2} \cdot\left(n^{2} / \lambda+n \lambda\right)\right)$.

(b) The maximum-cardinality bipartite matching problem can be solved in time $O\left(n^{2.5} / \log n\right)$ by a conservative algorithm.

2.6. Transitive Closure of Acyclic Graphs. In this section we discuss the computation of transitive closures. We restrict ourselves to acyclic (directed) graphs because acyclicity makes the problem more difficult; for general graphs the strongly connected components can always be computed first and then shrunk to obtain an acyclic graph. We assume

procedure search_path(node $v$ );

$\left({ }^{*}\right.$ when search_path $(v)$ is called, the recursion stack contains a path from a free node in $A$ to $v$ which is disjoint from the paths in $L$. The call either finds a path from $v$ to a free node in $B$ and then returns this path or, otherwise, returns nil *)

$w \leftarrow 0 ; P \leftarrow$ nil;

while $P=$ nil and $w<n \quad(\diamond)$

do if $\neg$ reached $[w]$ and $D[v, w] \quad(\diamond)$

then if $w$ is free

then $P \leftarrow((v, w))$

else $P \leftarrow$ search_path $(w)$;

if $P \neq$ nil then P.append $((v, w))$ fi

fi

$\operatorname{reached}[w] \leftarrow 1$;

fi

$w \leftarrow w+1(\diamond)$

od

return $P$

end

Program 6. Procedure search_path. 
our graphs to be topologically sorted, i.e., $V=\{0, \ldots, n-1\}$ and $(v, w) \in E$ implies $v<w$. The transitive closure $E^{*}$ of $E$ consists of all pairs $(v, w)$ such that there is a path from $v$ to $w$ using only edges in $E$. The transitive reduction $E_{\text {red }}$ of $E$ consists of all edges $(v, w) \in E$ such that there is no path of length at least two from $v$ to $w$. Let $m_{\text {red }}=\left|E_{\text {red }}\right|$.

Goralcikova and Koubek [GK] have shown how to compute $E^{*}$ in time $O\left(m_{\text {red }} \cdot n\right)$. The algorithm is quite simple. It steps through the vertices of $G$ in decreasing order. When vertex $v$ is considered it first initializes $E^{*}[v, v]=1$ and $E^{*}[v, w]=0$ for $w \neq v$, and then considers the edges $(v, w) \in E$ in increasing order of $w$. When $(v, w) \in E$ is considered, and $E^{*}[v, w]=0$ at that time, then $E^{*}[v, *] \leftarrow E^{*}[v, *] \vee E^{*}[w, *]$ is performed.

The crucial observation is that the OR of the $v$ th and the $w$ th row of $E^{*}$ is computed precisely for the edges $(v, w) \in E_{\text {red }}$; this implies the $O\left(m_{\text {red }} \cdot n\right)$ time bound. Therefore, if the $\lambda$-compression of $E^{*}$ is computed instead, then the time bound reduces to $O\left(n^{2}+m_{\text {red }} \cdot n / \lambda\right)$ where the $n^{2}$ term accounts for the computation of $\diamond E$ from $E$ and of $E^{*}$ from $\diamond E^{*}$.

LEMMA 4. On a $\lambda$-RAC the transitive closure of an n-vertex graph can be computed in time $O\left(n^{2}+m_{\text {red }} n / \lambda\right)$.

A random acyclic digraph is defined as follows. Let $\varepsilon, 0<\varepsilon<1$, be a fixed real number. For $v<w, \operatorname{prob}((v, w) \in E)=\varepsilon$, and the different events $(v, w) \in E$ are independent.

LEMMA 5 [Si]. $\quad E\left(m_{\text {red }}\right) \leq 2 n \log n$ for all $\varepsilon, 0<\varepsilon<1$.

THEOREM 5. The transitive closure of an acyclic digraph can be computed by a deterministic and conservative algorithm whose expected running time on the class of random. acyclic digraphs is $O\left(n^{2}\right)$.

PROOF. This is a direct consequence of the two preceding lemmas.

We compared the algorithm described above with the transitive closure algorithm of LEDA [MN]. The algorithm in LEDA computes the strongly connected components using the algorithm of Section 2.4, shrinks the components to obtain an acyclic graph, computes the transitive closure of the acyclic graph by means of the algorithm of Simon [Si] (this algorithm is also described in Section IV.3 of [M]), and finally translates the result back to the input graph. We modified the third step of the algorithm by using the algorithm of Goralcikova and Koubek [GK] together with bit-compression and then compared the running times of the two implementations on a SPARC workstation with 32 bit words. For random graphs and random acyclic graphs the running times of the two implementations are about the same (within $10 \%$ of each other). For graphs with $m_{r e d}=m=\Omega\left(n^{2}\right)$, e.g., graphs with three groups of $\Omega(n)$ nodes each and edges from each vertex in the first group to each vertex in the second group and from each vertex in the second group to each vertex in the third group, the bit-compression technique 
led to significant savings in running time. For $n=400$ we measured an improvement of about four. Also, on the same input the Goralcikova-Koubek algorithm without bitcompression is about eight times slower than the algorithm with bit-compression.

\section{Network Algorithms}

3.1. An $O\left(n^{2}(\log C / \log n)\right)$ Shortest-Path Algorithm. Let $N=(V, E, c, s)$ be an edge-weighted network with source $s$, i.e., $(V, E)$ is a directed graph, $c: E \rightarrow\{0, \ldots, C\}$ is an integer-valued cost function on the edges and $s \in V$ is a distinguished vertex. The goal is to compute arrays dist and pred, where, for all $v \in V$, dist $[v]$ is the length of a shortest path from $s$ to $v$ and pred $[v]$ is the predecessor of $v$ on a shortest path.

We solve the shortest-path problem in two phases; in the first phase we compute the dist-array and in the second phase we compute the pred-array. Both phases are based on Dijkstra's algorithm [D1]. We use two data structures, namely an integer $d$ and an array $Q: V \rightarrow\{-1,0, \ldots, C, \infty\}$ that serves as a priority queue. Assume that, for any $x \in\{0, \ldots, C\}$, we have $-1-x=-1$ and $\infty-x=\infty$. During the execution, a vertex $v$ is in one of two states; scanned $(Q[v]=-1$ ) or unscanned $(Q[v] \in\{0,1, \ldots, C\} \cup\{\infty\})$. We maintain the invariant that the distance to every scanned vertex from $s$ has been correctly computed, and that, for every unscanned vertex $v, Q[v]+d$ is the length of a shortest path from $s$ to $v$, subject to the restriction that every vertex in the path (except $v$ ) is scanned. In each iteration an unscanned vertex $v$ with minimal value $Q[v]$ is selected. Then dist $[v]=d+Q[v]$ according to the invariant. For later use in Phase 2 the value distmod $[v]=\operatorname{dist}[v] \bmod (C+1)$ is also stored. Also, $d$ is increased by $Q[v], Q[w]$ is decreased by $Q[v]$ for all unscanned vertices $w$, and all edges emanating from $v$ are inspected and cheaper paths are recorded. The details are given in Program 7. The proof of correctness is standard, see, for example, Section IV.7.2 of [M]. Note that all values computed are bounded by $C n=2^{\log n+\log C}$ and hence require $O(\lceil\log C / \log n\rceil)$ digits in base $2^{\lfloor\log n\rfloor}$. Hence Dijkstra's algorithm can be made to run in time $O\left(n^{2}\lceil\log C / \log n\rceil\right)$ by representing all $Q$ - and dist-values in base $2^{\lfloor\log n\rfloor}$. If $\lceil\log (3+C)\rceil>(\log n) / 3$, then our main claim is established: the shortest-paths problem can be solved in time $O\left(n^{2} \log C / \log n\right)$.

For the remainder of this subsection assume that $\lceil\log (3+C)\rceil \leq(\log n) / 3$. We show how to achieve a running time of $O\left(n^{2} \log C / \log n\right)$. Let $b=\lceil\log (3+C)\rceil$ and let $t \in \mathbb{N}$ be such that $t \cdot b \leq \log n$. The exact value of $t$ will be specified later. Also interpret $c$ as a $V \times V$ matrix with entries in $\{0, \ldots, C\}$. We partition $Q$ and each row of $c$ into blocks of length $t$ and represent each block as a single integer. More precisely, for $a \in D:=\{-1,0, \ldots, C, \infty\}$ let

$$
\hat{a}= \begin{cases}C+2 & \text { if } a=-1, \\ C+1 & \text { if } a=\infty \\ a & \text { if } 0 \leq a \leq C\end{cases}
$$

and for $a_{0}, \ldots, a_{t-1} \in D$ let $\operatorname{Comp}\left(a_{0}, \ldots, a_{t-1}\right)=\sum_{0 \leq i<t} \hat{a}_{i}(C+3)^{i}$. Then clearly $0 \leq \operatorname{Comp}\left(a_{0}, \ldots, a_{t-1}\right)<(C+3)^{t} \leq 2^{b t} \leq n$. The $t$-compressed representation of a sequence $a_{0}, \ldots, a_{n-1}$ of values in $D$, where for simplicity we assume $t$ to be a divisor of 
(1) $d \leftarrow Q[s] \leftarrow 0$

(2) for all $v \neq s$ do $Q[v] \leftarrow \infty$ od;

(3) while $\exists$ unscanned vertex

(4) do let $v$ be an unscanned vertex with minimal entry $Q[v]$;

(5) $d \leftarrow \operatorname{dist}[v] \leftarrow d+Q[v]$; distmod $[v] \leftarrow d \bmod (C+1)$;

(6) for all unscanned $w$

(7) do $Q[w] \leftarrow Q[w]-Q[v]$ od $\quad\left({ }^{*} \infty-Q[v]=\infty^{*}\right)$

(8) $\quad Q[v] \leftarrow-1 ; \quad\left({ }^{*} v\right.$ is now scanned $\left.{ }^{*}\right)$

(9) for all unscanned $w$ with $(v, w) \in E$

(10) do if $c(v, w)<Q[w]$

(11) then $Q[w] \leftarrow c(v, w)$

(12) fi

(13) od

(14) od

Program 7. Shortest paths: phase 1.

$n$, is the sequence $\operatorname{Comp}\left(a_{0}, \ldots, a_{t-1}\right), \operatorname{Comp}\left(a_{t}, \ldots, a_{2 t-1}\right), \ldots$ We assume from now on that $c$ and $Q$ are available in $t$-compressed form and show next how the row-scans implicit in lines 4, 6,7, and 9-11 can be done in time $O(n / t)$ each.

For integers $A, B, \hat{a}$ with $0 \leq A, B \leq(C+3)^{t}-1,0 \leq \hat{a} \leq C+1, A=$ $\operatorname{Comp}\left(a_{0}, \ldots, a_{t-1}\right)$, and $B=\operatorname{Comp}\left(b_{0}, \ldots, b_{t-1}\right)$ let $\operatorname{select} \min (B)=(i, \hat{b})$ where $0 \leq i \leq t-1$ and $\hat{b}_{i}=\min \left(\hat{b}_{0}, \hat{b}_{1}, \ldots, \hat{b}_{t-1}\right)$, decrease $(A, \hat{a})=A^{\prime}$, where $A^{\prime}=$ $\operatorname{Comp}\left(a_{0}-a, \ldots, a_{t-1}-a\right)$ if $0 \leq \hat{a} \leq C$, and $A^{\prime}=A$ otherwise, and componentwise $\min (A, B)=A^{\prime}$ where $A^{\prime}=\operatorname{Comp}\left(a_{0}^{\prime}, \ldots, a_{t-1}^{\prime}\right)$ and

$$
a_{i}^{\prime}= \begin{cases}b_{i}, & \text { if } b_{i}<a_{i} \leq C+1, \\ a_{i} & \text { otherwise. }\end{cases}
$$

\section{LEMMA 6.}

(a) The function tables for functions select_min, decrease, and componentwise_min can be computed in time $O\left(t \cdot 2^{2 b t}\right)$.

(b) Given tables for functions select_min, decrease, and componentwise_min, phase 1 runs in time $O\left(n^{2} / t\right)$.

PROOF. (a) The tables have at most $2^{b t}, 2^{b} \cdot 2^{b t}$, and $2^{2 b t}$ entries, respectively. Each entry can be computed in time $O(t)$.

(b) Each execution of lines 4, 6, 7, and 9-11 is tantamount to $n / t$ evaluations of functions select_min, decrease, and componentwise_min, respectively, and each evaluation takes constant time by table look-up. Finally, an execution of line 5 takes time $O(\log n / \log C)=O(\log n)$ since $0 \leq d \leq n C$, and an execution of line 8 takes constant time using an appropriate table. Also, initialization takes linear time $O(n)$.

LEMMA 7. Let $t=\lfloor(\log n) / 3 b\rfloor$. Given the distance matrix $c$ in $t$-compressed form the 
shortest distances from a given vertex $s$ in an n-vertex graph can be computed in time $O\left(n^{2}(\log \max (2, C)) / \log n\right)$.

PROOF. For $t=\lfloor(\log n) / 3 b\rfloor$ we have $O\left(t \cdot 2^{2 b t}\right)=O(n \log n)$ and $O\left(n^{2} / t\right)=$ $O\left(n^{2}(\log \max (2, C)) / \log n\right)$. The claim now follows from the preceding lemma.

We next turn to the computation of the shortest-path tree. In the standard implementation, of Dijkstra's algorithm the pred-array is computed together with the dist-array by adding the assignment pred $[w] \leftarrow v$ in line 11 . We cannot do that here because each such assignment requires us to write $\log n$ bits and therefore several of these assignments cannot be compressed into a single assignment. We propose computing the predecessor information in a second phase. The program for the second phase is the same as for the first phase except that line 5 is replaced by

(5a) $d \bmod \leftarrow(d \bmod +Q[v]) \bmod (C+1)$

and line 11 is replaced by

(11a) $Q[w] \leftarrow c(v ; w)$

(11b) if $(\operatorname{dmod}+Q[w]) \bmod (C+1)=\operatorname{distmod}[w]$

(11c) then pred $[w] \leftarrow v$ fi

LEMMA 8. Phase 2 computes the pred-array correctly. Also, given a t-compressed $c$ for $t=\lfloor(\log n) / 3 b\rfloor$ it can be made to run in time $O\left(n^{2}(\log \max (2, C)) / \log n\right)$.

Proof. We first prove correctness. Phase 1 computes distmod $[w]=\operatorname{dist}[w] \bmod$ $(C+1)$ for all vertices $w$. Also, dist $[w] \leq d+Q[w]$ and $d \leq$ dist $[w]$ for all unscanned nodes $w$, and $d \bmod =d \bmod (C+1)$ throughout execution of phase 2 . Thus, whenever line 11a is executed we have $d \leq \operatorname{dist}[w] \leq d+Q[w] \leq d+C$ and hence whenever line $11 \mathrm{c}$ is executed we have $\operatorname{dist}[w]=d+Q[w]$ and thus all assignments in line 11c are valid. Next consider for any vertex $w \neq s$ the last assignment to $Q[w]$ in line 11a. At this point, we have $d+Q[w]=\operatorname{dist}[w]$ and hence there will be an assignment to pred $[w]$. Finally observe that for any unscanned vertex $w$ the value $d+Q[w]$ never increases, and decreases in every execution of line $11 \mathrm{a}$. Thus there will be at most one assignment to pred $[w]$. This proves correctness and also that line $11 \mathrm{c}$ is executed at most $n$ times.

We next turn to the running time. For $0 \leq A, B, D \leq(C+3)^{t}-1, A=\operatorname{Comp}\left(a_{0}, \ldots\right.$, $\left.a_{t-1}\right), B=\operatorname{Comp}\left(b_{0}, \ldots, b_{t-1}\right), D=\operatorname{Comp}\left(d_{0}, \ldots, d_{t-1}\right)$, and $0 \leq d \leq C$ let ext_min $(A, B, d, D)=\left(A^{\prime}, k\right)$ where $A^{\prime}=\operatorname{Comp}\left(a_{0}^{\prime}, \ldots, a_{t-1}^{\prime}\right)$ is defined as in the case of componentwise $\min (A, B), k=\sum_{0 \leq i<t} k_{i} 2^{i}$, and

$$
k_{i}= \begin{cases}1 & \text { if } a_{i}^{\prime}=b_{i}<a_{i} \text { and } d+b_{i}=d_{i} \bmod (C+1), \\ 0 & \text { otherwise }\end{cases}
$$

We can use function ext_min in lines $11 \mathrm{a}-\mathrm{c}$ as follows: For $t$ vertices $v_{0}, \ldots, v_{t-1}$ let $A=\operatorname{Comp}\left(Q\left[v_{0}\right], \ldots, Q\left[v_{t-1}\right]\right), B=\operatorname{Comp}\left(c\left[v, v_{0}\right], \ldots, c\left[v, v_{t-1}\right]\right)$, and $D=$ Comp $\left(\right.$ distmod $\left[v_{0}\right], \ldots$, distmod $\left.\left[v_{t-1}\right]\right)$. Then $\operatorname{ext} \min (A, B, \operatorname{dmod}, D)=\left(A^{\prime}, k\right)$ where 
$A^{\prime}$ is the new content of $Q$ and $k$ codes all indices for which line $11 \mathrm{c}$ has to be executed. Thus lines $11 \mathrm{a}-\mathrm{c}$ can be executed for $t$ vertices in time $O(1)$ plus the number of executions of line $11 \mathrm{c}$, which amounts to $O\left(n^{2} / t+n\right)$ overall. Finally, a table for ext_min can be computed in time $O\left(t \cdot 2^{b} \cdot 2^{3 b t}\right)=O\left(n^{4 / 3}(\log n)^{2}\right)$.

THEOREM 6. Let $b=\lceil\log (3+C)\rceil \leq(\log n) / 3$ and $t=\lfloor\log n / 3 b\rfloor$. Given the distance matrix $c$ in $t$-compressed form the shortest-path problem on an $n$-vertex graph with edge weights in $\{0, \ldots, C\} \cup\{\infty\}$ can be solved in $O\left(n^{2} \log \max (2, C) / \log n\right)$ time.

ProOF. Obvious from Lemmas 7 and 8.

3.2. The Uncapacitated Transportation Problem. Let $(V, W, E)$ be a symmetric directed bipartite graph, i.e., $E \subseteq(V \times W) \cup(W \times V)$ and $(v, w) \in E$ iff $(w, v) \in E$, let $b: V \cup W \rightarrow[-U \cdots U]$ be a supply-demand function on the vertices $\left(\sum_{x \in V \cup W} b(x)=\right.$ $0)$, and let $c: E \rightarrow[-C \cdots C]$ be a cost function on the edges $(c(v, w)=-c(w, v)$ and $c(v, w) \geq 0$ for $(v, w) \in E \cap(V \times W))$. Let cap: $E \rightarrow\{0, \infty\}$ with $\operatorname{cap}(v, w)=\infty$ and $\operatorname{cap}(w, v)=0$ for $(v, w) \in E \cap(V \times W)$ be a capacity function on the edges. A solution for the transportation problem $(V, W, E, b, c)$ is an integer-valued function $f$ on the edges such that:

(1) $f(x, y)=-f(y, x)$ and $f(x, y) \leq \operatorname{cap}(x, y)$, for all $(x, y) \in E$.

(2) $b(x)=\sum_{(x, y) \in E} f(x, y)$, for all $x \in V \cup W$.

(3) $\operatorname{cost}(f)=\sum_{f(x, y) \geq 0} f(x, y) \cdot c(x, y)$ is minimized.

Ahuja et al. [AGOT] have shown how to solve the uncapacitated transportation problem in time $O\left(\left(n m+n^{2} \log U\right) \log n C\right)$; see also [AMO]. We take the latter paper as the basis of our exposition. We need the following definitions. A function $f$ satisfying (1) is called a pseudoflow. The imbalance of a vertex $x$ with respect to a pseudoflow $f$ is given by $\operatorname{imb}(x)=b(x)-\sum_{(x, y) \in E} f(x, y)$, and the residual capacity of an edge $(x, y)$ is given by $\operatorname{rescap}(x, y)=c a p(x, y)-f(x, y)$. A dual function $\pi$ is any function $\pi: V \cup W \rightarrow R$. A pseudoflow $f$ is $\varepsilon$-optimal with respect to real number $\varepsilon \geq 0$ and dual function $\pi$ if $\vec{c}(x, y):=c(x, y)+\pi(x)-\pi(y) \geq-\varepsilon$ for all $(x, y) \in E$ with rescap $(x, y)>0 ; \bar{c}(x, y)$ is called the reduced cost of edge $(x, y)$. A pseudoflow is a flow if it satisfies (1) and (2).

\section{FACT 1.}

(a) Let $\varepsilon \leq 1 / n$ and let $f$ be aflow which is $\varepsilon$-optimal with respect to some dual function $\pi$. Then $f$ is optimal.

(b) Let $f$ be any flow and let $\pi(x)=0$ for all $x \in V \cup W$. Then $f$ is $C$-optimal with respect to $\pi$.

Ahuja $e t$ al. solve the uncapacitated transportation problem by $\log n C$ iterations of a procedure improve_approximation. This procedure takes as input a dual function $\pi^{\prime}$ and an $\varepsilon>0$ and returns a flow $f$ and a dual function $\pi$ such that $f$ is $(\varepsilon / 2)$-optimal with respect to $\pi$. The procedure requires the precondition that there is an $\varepsilon$-optimal flow $f^{\prime}$ with respect to $\pi^{\prime}$, although it need not know this flow. For $\varepsilon=C$, the constant 


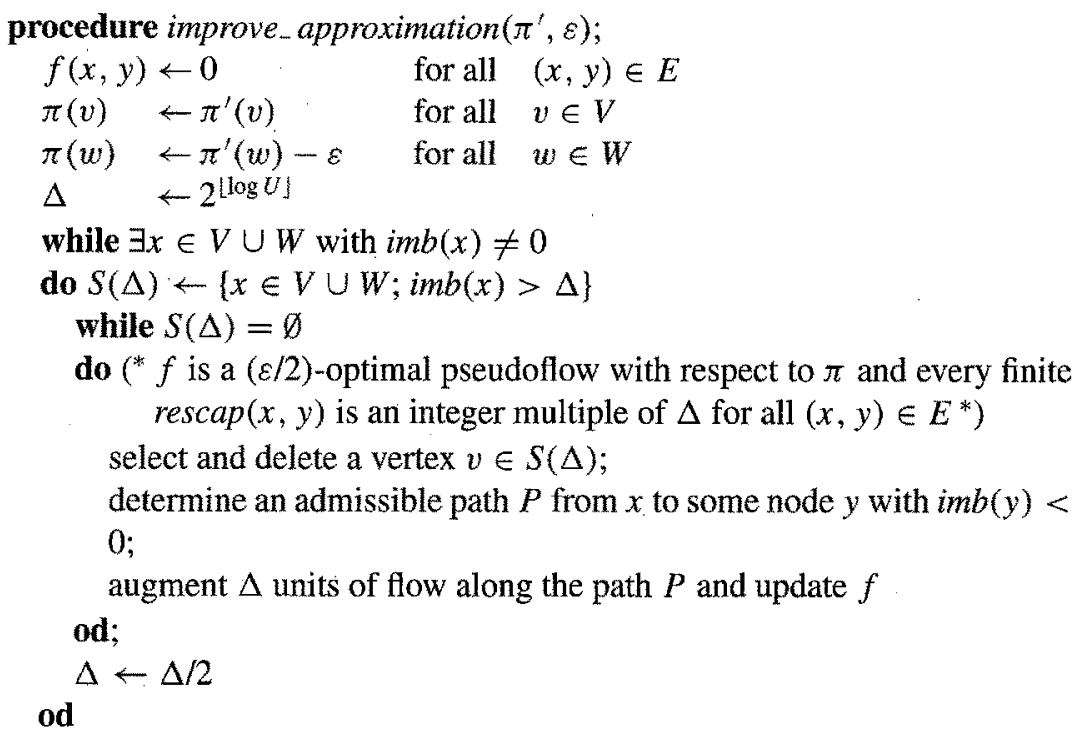

Program 8. Going from $\varepsilon$-optimality to $(\varepsilon / 2)$-optimality.

zero dual function has this property by part (b) of Fact 1. After $\log n C$ applications of improve_approximation an (1/n)-optimal flow is obtained. It is optimal according to part (a) of Fact 1. The procedure improve, approximation (see Program 8) starts with the pseudoflow $f(x, y)=0$ for all $(x, y) \in E$ and a dual function $\pi$ such that $f$ is $(\varepsilon / 2)$-optimal (in fact, 0 -optimal) with respect to $\pi$. It then turns $f$ into a flow by successive augmentations along so-called admissible paths. An admissible path consists of admissible edges and leads from a vertex $x$ with positive imbalance to a vertex $y$ with negative imbalance. An edge is admissible, if its residual capacity is positive and its reduced cost is negative. An admissible path starting in a vertex $x$ (with $\operatorname{im} b(x)>0$ ) is constructed iteratively. Suppose that we already have an admissible path from $x$ to some other node $y$. If $i m b(y)<0$, then we are finished. If $i m b(y) \geq 0$ and there is an admissible edge $(y, z)$, then $z$ is added to $P$ (advance). If there is no such edge, then $\pi(y)$ is decreased by $\varepsilon / 2$ and $y$ (if different from $x$ ) is removed from $P$ (retreat).

FACT 2. Improve approximation executes $O\left(n^{2} \log U\right)$ advance and retreat steps and runs in time $O\left(n^{2} \log U\right)$ plus the time needed to identify admissible edges. Moreover, $\pi(x), x \in V \cup W$, is changed only $O(n)$ times within a call of improve approximation.

The $O(n m)$ term in the running time of the Ahuja et al. algorithm results from the fact that the adjacency list of each vertex $v$ is scanned $O(n)$ times, once for each change of the dual value $\pi(v)$. We now discuss how to speed up the search for admissible edges in dense graphs.

Define the truncated reduced cost $\tilde{c}(x, y)$ of an edge $(x, y) \in E$ by

$$
\tilde{c}(x, y)=\left\lfloor\frac{\bar{c}(x, y)}{(\varepsilon / 2)}\right\rfloor .
$$


LEMMA 9. $(x, y) \in E$ is admissible iff $\tilde{c}(x, y)=-1$ and rescap $(x, y)>0$.

PROOF. $\quad(\Leftarrow)$ If $\tilde{c}(x, y)=-1$, then $\bar{c}(x, y)<0$.

$\Leftrightarrow$ If $(x, y)$ is admissible, then $\bar{c}(x, y)<0$ and $\operatorname{rescap}(x, y)>0$. By $(\varepsilon / 2)$ optimality, we also have $\vec{c}(x, y) \geq-\varepsilon / 2$. Thus $\tilde{c}(x, y)=-1$.

We maintain a matrix $D$ which approximates $\tilde{c}$. We have $D[x, y] \in[-z \cdots z] \cup$ $\{-\infty, \infty\}$, where $z$ is a constant to be fixed later, and $D[x, y]=-1$ iff $(x, y) \in E$ and $\tilde{c}(x, y)=-1$. Let $b \geq\lceil\log (2 z+3)\rceil$, i.e., $b$ bits suffice to encode an entry of $D$, and let $t \in \mathbb{N}$ be such that $t^{2} b \leq \log n$. We partition $D$ into $t \times t$ submatrices and store each submatrix in a single RAC-word.

The invariant $D[x, y]=-1$ iff $\tilde{c}(x, y)=-1$ is maintained by updating the entries in row and column $x$ after every $z / 2$ changes of $x$ 's dual value, i.e., $D[x, y]$ is set to $\tilde{c}(x, y)$ if $(x, y) \in E$ and $\tilde{c}(x, y) \in[-z, z]$, and to $+\infty$ or $-\infty$ otherwise. This takes time $O(n)$ for each update and hence time $O\left(n^{3} / z\right)$ in total.

For the search for admissible edges we also maintain a (compressed) matrix $R$ with $R[x, y] \in\{0,1\}$ and $R[x, y]=1$ iff $\operatorname{rescap}(x, y)>0 . R[x, y]$ can be updated in time $O(1)$ per change of the preflow $f$ and hence in time $O\left(n^{2} \log U\right)$ totally. Also, given appropriate tables, a scan of an adjacency list takes time $O(n / t)$ plus the number of admissible edges found. The total time for scanning adjacency lists is therefore $O\left(n^{3} / t+n^{2} \log U\right)$. Finally, the tables required can be precomputed in time $O\left(2^{2 t^{2} b}\right)$.

With the choice $t=z=\left\lfloor(\log n / \log \log n)^{1 / 2}\right\rfloor$, we obtain a running time of

$$
O\left(n^{3}\left(\frac{\log \log n}{\log n}\right)^{1 / 2}+n^{2} \log U\right)
$$

for each call of improve_approximation. We summarize in:

THEOREM 7. The uncapacitated transportation problem $(V, W, E, b, c)$ with $b$ and $c$ integral, $|V|=|W|=n$, absolute values of supplies and demands bounded by $U$, and absolute values of costs bounded by $C$ can be solved in time

$$
O\left(\left(n^{3}\left(\frac{\log \log n}{\log n}\right)^{1 / 2}+n^{2} \log U\right) \log n C\right) .
$$

3.3. The Assignment Problem. Let $(U, W, E)$ be a bipartite graph and let $c: E \rightarrow$ $[0 . . C]$ be a cost function on the edges. We assume that the edge costs are nonnegative integers; also, we assume that the graph has a perfect matching, since this can be checked using the algorithm in Section 2.5. Let $n$ denote $|U|=|W|$. A solution to the assignment problem is a perfect matching $M$ that maximizes $c(M)=\sum_{u w \in M} c(u w)$; throughout this section we use $u w$ to denote the unordered edge $\{u, w\}$. We show how to implement a variant of the $O\left(n^{2,5} \log n C\right)$ algorithm of Orlin and Ahuja [OA] so that it runs in time $O\left(n^{2.5} \log n C \cdot(\log \log n / \log n)^{1 / 4}\right)$. The same speed-up can also be obtained for the Gabow and Tarjan [GT] assignment algorithm.

As most assignment algorithms do, the Orlin and Ahuja algorithm computes not only a perfect matching of maximum cost, but also a near-optimal dual solution $y: U \cup W \rightarrow Z$. 
A dual solution is required to be dominating with respect to the cost function $c$, i.e.,

$$
y(u)+y(w) \geq c(u w), \quad \forall u w \in E .
$$

(Indeed, if we formulate the assignment problem as a linear program and write down the dual, then the dual variables are $y(v), v \in U \cup W$, and the dual constraints are that $y$ be dominating.) An edge $u w$ is called tight with respect to $y$ if $y(u)+y(w)=c(u w)$.

FACT 3. A perfect matching $M$ has maximum cost if there is a dominating dual solution $y$ such that every edge in $M$ is tight.

The fact follows since every edge of $M$ is tight, so $c(M)=\sum_{u} y(u)+\sum_{w} y(w)$; moreover, for every matching $M^{\prime}$ each edge $u^{\prime} w^{\prime} \in M^{\prime}$ has $c\left(u^{\prime} w^{\prime}\right) \leq y\left(u^{\prime}\right)+y\left(w^{\prime}\right)$, so $c\left(M^{\prime}\right) \leq \sum_{u} y(u)+\sum_{w} y(w)$.

For a constant $\alpha>0$, a dominating dual solution $y$ is called $\alpha$-tight with respect to $c$ if a perfect matching $M$ exists such that every edge $u w$ in $M$ is $\alpha$-tight, i.e.,

$$
y(u)+y(w) \leq c(u w)+\alpha, \quad \forall u w \in M .
$$

(If $M$ is known, then we say that $y$ is $\alpha$-tight with respect to $c$ and $M$.) The dual solution $(y(u)=0, \forall u \in U, y(w)=C, \forall w \in W)$, where $C \geq \max _{u w \in E} c(u w)$, is dominating and $C$-tight with respect to $c$.

LEMMA 10 [B]. Let $M$ be a perfect matching such that there is a dominating dual solution $y$ which is $\alpha$-tight with respect to $c$ and $M$.

(i) For every perfect matching $M^{\prime}, c\left(M^{\prime}\right) \leq c(M)+\alpha n$.

(ii) If there is an integer $k, k>\alpha n$, such that every edge cost $c(u w)$ is an integer multiple of $k$, then $M$ is a matching of maximum cost.

Proof. Part (i) follows since

$$
c\left(M^{\prime}\right)=\sum_{u w \in M^{\prime}} c(u w) \leq \sum_{u} y(u)+\sum_{w} y(w) \leq \sum_{u w \in M}(c(u w)+\alpha)=c(M)+\alpha n .
$$

Part (ii) follows since, for every perfect matching $M^{\prime}, c\left(M^{\prime}\right)$ is an integer multiple of $k>\alpha n$, but then by part (i) no $M^{\prime}$ with $c\left(M^{\prime}\right)>c(M)$ exists.

A high-level description of the assignment algorithm is given in Program 9. The algorithm works iteratively, scaling the cost function in every iteration. If every edge has zero cost, then the algorithm returns an arbitrary perfect matching by using the algorithm of Section 2.5. Otherwise, the algorithm scales the edge costs $c(u w)$ to $\lfloor c(u w) / 2\rfloor$, and recursively solves the smaller problem, finding a dominating dual solution $\tilde{y}$ and a matching $\tilde{M}$ such that $\tilde{y}$ is 1 -tight with respect to $\lfloor c(u w) / 2\rfloor$ and $\widetilde{M}$. We obtain a dominating dual solution $y^{\prime}$ with respect to the original costs $c$ by taking $y^{\prime}(u)=2 \tilde{y}(u)$ for each $u \in U$, and taking $y^{\prime}(w)=1+2 \tilde{y}(w)$ for each $w \in W$. Then a combination of Bertsekas's auction algorithm [B] and the shortest augmenting paths algorithm is used with inputs $y^{\prime}$, the original costs $c$, and graph $(U, W, E)$ to find a new dominating dual 
procedure assignment $(U, W, E, c)$, returns $(M, y)$;

precondition: $(U, W, E)$ has a perfect matching, and $c(u w) \geq 0$ for all $u w \in E$.

postcondition: $M$ is a perfect matching and $y:(U \cup W) \rightarrow Z$ is a dominating dual solution that is 1-tight with respect to $c$ and $M$.

begin if $c(u w)=0$ for all $u w \in E$

then return $(M, y)$ where $M$ is any perfect matching, and $(y(u)=0$, $\forall u \in U, y(w)=0, \forall w \in W)$

else $\quad$ let $\tilde{c}(u w)=\lfloor c(u w) / 2\rfloor$ for all $u w \in E$;

$(\tilde{M}, \tilde{y}) \leftarrow \operatorname{assignment}(U, W, E, \tilde{c})$;

let $y^{\prime}(u)=2 \tilde{y}(u), \forall u \in U$, and $y^{\prime}(w)=1+2 \tilde{y}(w), \forall w \in W$;

$\left({ }^{*} y^{\prime}\right.$ is dominating and 3-tight with respect to original $\operatorname{costs} c^{*}$ )

$(M, y) \leftarrow \operatorname{auction}\left(U, W, E, c, y^{\prime}\right)$;

( ${ }^{*} y$ is dominating and 1-tight with respect to $M^{*}$ )

return $(M, y)$;

fi

end

Program 9. The assignment algorithm.

solution $y$ and a new perfect matching $M$ such that $y$ is 1-tight with respect to $c$ and $M$. The above subroutine, which we call auction, turns out to be quite efficient because the starting dual solution $y^{\prime}$ is nearly optimal: Lemma 11 shows that $y^{\prime}$ is 3-tight with respect to the original costs $c$.

LEMMA 11. The dual solution $y^{\prime}$ is dominating and 3-tight with respect to the original $\operatorname{costs} c$.

PROOF. Since $\tilde{y}$ is dominating and 1-tight with respect to $\tilde{c}$ and $\tilde{M}$, for every edge $u w \in E$,

$$
y^{\prime}(u)+y^{\prime}(w)=2(\tilde{y}(u)+\tilde{y}(w))+1 \geq 2 \tilde{c}(u w)+1 \geq c(u w)
$$

moreover, if $u w \in \tilde{M}$, then

$$
y^{\prime}(u)+y^{\prime}(w)=2(\tilde{y}(u)+\tilde{y}(w))+1 \leq 2(\tilde{c}(u w)+1)+1 \leq c(u w)+3 .
$$

LEMMA 12 [OA]. Let $0 \leq c(u w) \leq C$ for all $u w \in E$. A call assignment $(U, W, E$, $(n+1) \cdot c)$ returns an optimal matching and runs in time $O\left(\left(T_{\text {auction }}+n\right) \log n C+\right.$ $\left.n^{2.5} / \log n\right)$, where $T_{\text {auction }}$ is the time complexity of a call of the auction subroutine.

Assume that the procedure auction returns $M, y$ such that $y$ is 1-tight with respect to $(n+1) \cdot c$ and $M$. Then the above lemma follows from Lemma 10 , and the fact that the depth of recursion in our assignment algorithm is $\log n C$, where each level of recursion has time complexity $O\left(T_{\text {auction }}+n\right)$. If every edge $u w$ has $c(u w)=0$, then a perfect matching can be found in time $O\left(n^{2.5} / \log n\right)$, according to Section 2.5. 
The procedure auction is defined by Program $10 ; \gamma \geq 1$ is a constant to be fixed later. We use $U_{\text {free }}\left(W_{\text {free }}\right)$ to denote the set of free vertices in $U(W)$ with respect to the current matching $M$, i.e., $U_{\text {free }}=\{u \in U ; \exists u w \in M\}$. Auction consists of two phases. The first phase uses Bertsekas's bidding heuristic to find quickly a "1-tight" (not necessarily perfect) matching $M$ such that few nodes are free with respect to $M$. The second phase repeatedly uses the classical Hungarian search to adjust dual variables and find " 1 -tight" augmenting paths, and augments $M$ using these paths.

For ease of description of phase 1, we transform the edge costs to $\bar{c}(u w)=c(u w)-$ $y^{\prime}(u)-y^{\prime}(w), \forall u w \in E$, where $y^{\prime}$ is the initial dual solution, and we introduce a new dual solution $z:(U \cup W) \rightarrow Z$ that is dominating with respect to $\bar{c}$. Since $y^{\prime}$ is dominating with respect to $c$, we have $\bar{c}(u w) \leq 0, \forall u w \in E$. Initially, the matching $M$ is empty, and $z(v)=0, \forall v \in U \cup W$. In each iteration of bidding, we choose a $u \in U$ with sufficiently large $z(u)$ and either decrease $z(u)$ or add a "tight" edge $u w$ to $M$. In the latter case, we immediately increase $z(w)$ by one, since edges in $M$ are allowed to be "1-tight." An important point is that there are only $O(\sqrt{n / \lambda})$ free vertices with respect to $M$ when phase 1 terminates (Lemma 13(c)).

In phase 2 we revert back to the originals $\operatorname{costs} c$, and combine the two dual solutions $y^{\prime}$ and $z$ to obtain a dual solution $y$ that is dominating with respect to $c$. Moreover, $y$ is near optimal in the sense that the gap $g=\sum_{u} y(u)+\sum_{w} y(w)-c\left(M^{*}\right)$, where $M^{*}$ is an optimal assignment, is $\leq 4 n$. Call an edge $u w$ eligible with respect to the current $y$ if $y(u)+y(w) \leq c(u w)+1$. To augment $M$ to a perfect matching consisting of eligible edges, we repeatedly find an augmenting path $P$ of eligible edges by applying the Hungarian search: Using only the eligible edges, we construct an alternating forest $F$ whose root nodes are the nodes $w, w \in W_{\text {free. }}$. If $F$ contains a node $u, u \in U_{\text {free }}$, then we have the desired augmenting path. Otherwise, we repeatedly adjust $y$ and extend $F$ until $F$ contains a node $u, u \in U_{\text {free }}$. To adjust $y$, we compute

$$
\delta=\min \{y(u)+y(w)-c(u w): w \in W \cap V(F) \text { and } u \in U-V(F)\} \text {. }
$$

For each $w \in W \cap V(F)$, we decrease $y(w)$ by $\delta$, and for each $u \in U \cap V(F)$, we increase $y(u)$ by $\delta$. Clearly, eligible edges with both ends in $F$ stay eligible after adjusting $y$; moreover, at least one edge $u w, w \in W \cap V(F), u \in U-V(F)$, that was not eligible before adjusting $y$ becomes eligible. We extend $F$ by adding $u w$ and $u$. Consider the overall time complexity of computing $\delta$ between two consecutive augmentations of $M$. By using a heap to store an appropriate key for each $u \in U-V(F)$, a bound of $O(|E| \log n)$ is easily achieved. However, this can be improved to $O(|E|+n)$ by using a "bucket-based" data structure (as in Dial's implementation of Dijkstra's algorithm). This follows since every adjustment of $y$ by $\delta$ decreases $Y=\sum_{u} y(u)+\sum_{w} y(w)$ by $\delta\left|W_{\text {free }}\right|$, and $Y$ decreases by at most the gap $g \leq 4 n$, hence, $\sum \delta$ over all adjustments of $y$ between two consecutive augmentations of $M$ is at most $4 n$. See Section 2.1 of [GT] for details.

\section{LEMMA 13. In the procedure auction:}

(a) (i) Initially, $z$ is dominating and 3-tight with respect to $\vec{c}$.

(ii) Throughout phase $1, z$ is dominating with respect to $\bar{c}$, and, for every edge $u w \in M, z(u)+z(w) \leq \bar{c}(u w)+1$. 
procedure auction $\left(U, W, E, c, y^{\prime}\right)$ returns $(M, y)$;

precondition: $y^{\prime}$ is a dominating and 3-tight dual solution with respect to $c$; postcondition: $M$ is a perfect matching and $y:(U \cup W) \rightarrow Z$ is a dominating dual solution that is 1-tight with respect to $c$ and $M$.

\section{begin}

let $\bar{c}(u w)=c(u w)-y^{\prime}(u)-y^{\prime}(w)$ for all edges $u w \in E$;

$M \leftarrow \emptyset$;

let $(z(u)=0, \forall u \in U, z(w)=0, \forall w \in W)$

$\left({ }^{*} \bar{c}(u w) \leq 0, \forall u w \in E\right.$, and $z:(U \cup W) \rightarrow Z$ is a dominating dual solution with respect to $\bar{c}^{*}$ )

( ${ }^{*}$ Phase 1 : bidding*)

while $\exists u \in U_{\text {free }}: z(u) \geq-\lfloor\gamma \sqrt{n}\rfloor+1$

do let $u$ be such a vertex;

if $\exists w \in W: \bar{c}(u w)=z(u)+z(w)$

then let $w \in W$ be such a vertex;

if $w$ is matched by $M$ to $u^{\prime} \in U$ (i.e., $\exists u^{\prime}: u^{\prime} w \in M$ )

then $M \leftarrow M-\left\{u^{\prime} w\right\} \cup\{u w\}$;

fi

$z(w) \leftarrow z(w)+1$

fi

else $z(u) \leftarrow z(u)-1$;

od

let $\left(y(u)=y^{\prime}(u)+z(u), \forall u \in U, y(w)=y^{\prime}(w)+z(w), \forall w \in W\right)$;

( ${ }^{*} y$ is a dominating dual solution with respect to original costs $c$, for each $u w \in M, y(u)+y(w)=c(u w)+1$, and $\left|U_{\text {free }}\right|=\left|W_{\text {free }}\right| \leq 8 \sqrt{n} / \gamma$ (see Lemma 13) ${ }^{*}$ )

(*Phase 2: Hungarian search*)

while $\exists w \in W_{\text {free }}$

do (* call an edge $u w$ eligible if $y(u)+y(w) \leq c(u w)+1^{*}$ )

adjust the dual solution $y$ until an augmenting path $P$ with respect to $M$ is found such that each edge in $P$ is eligible;

$y$ is adjusted using the Hungarian search, such that $y$ stays dominating with respect to $c$, and every edge $u w$ in the current matching $M$ stays eligible;

augment $M$ along path $P$, i.e., replace $M$ by the symmetric difference of $M$ and $P$;

od

${ }^{*} M$ is a perfect matching, and $y$ is dominating and 1-tight with respect to $c$ and $M^{*}$ )

end

Program 10. The auction algonithm. 
(b) There are at most $2 \gamma n^{3 / 2}$ executions of the while-loop in phase 1 .

(c) $\left|U_{\text {free }}\right|=\left|W_{\text {free }}\right| \leq 8 \sqrt{n} / \gamma$ at the end of phase 1 .

(d) At the start of phase 2, $y$ is a dominating dual solution with respect to $c$ such that $\sum_{u} y(u)+\sum_{w} y(w)-c\left(M^{*}\right) \leq 4 n$, where $M^{*}$ is an optimal assignment.

(e) The total time complexity of phase 2 is $O\left(n^{2.5} / \gamma\right)$.

PROOF. (a) Initially, since $y^{\prime}$ is dominating and 3-tight with respect to $c,(z(v)=0$, $\forall v \in U \cup W$ ) is dominating and 3-tight with respect to $\bar{c}$. Part (ii) follows by induction on the number of steps in phase 1 (bidding).

(b) Clearly, $1-\lfloor\gamma \sqrt{n}\rfloor \leq z(u) \leq 0$ for every $u \in U$ and $z(w) \geq 0$ for all $w \in W$ throughout phase 1. Also, after every increase of $z(w), w \in W$, there is a vertex $u \in U$ such that $\bar{c}(u w)+1=z(u)+z(w)$. Thus $z(w) \leq \bar{c}(u w)+1-z(u) \leq 1+\lfloor\gamma \sqrt{n}\rfloor-1 \leq$ $\gamma \sqrt{n}$. Since each iteration of the while-loop either increments $z(w)$ for some $w \in W$ or decrements $z(u)$ for some $u \in U$, the bound follows.

(c) Let $M$ and $z$ be the matching and the dual solution (with respect to $\vec{c}$ ) at the end of phase 1 , respectively, and let $\bar{M}$ be an optimal assignment with respect to $\bar{c}$. Then

$$
\begin{aligned}
-3 n & \leq \bar{c}(\bar{M}) \\
& \leq \sum_{u \in U} z(u)+\sum_{w \in W} z(w) \\
& =\sum_{u w \in M}(z(u)+z(w))+\sum_{u \in U_{\text {free }}} z(u)+\sum_{w \in W_{\text {free }}} z(w) \\
& \leq \sum_{u w \in M}(\bar{c}(u w)+1)+\left|U_{\text {free }}\right|(-\lfloor\gamma \sqrt{n}\rfloor) \\
& \leq|M|-\left|U_{\text {free }}\right|(\lfloor\gamma \sqrt{n}\rfloor),
\end{aligned}
$$

where the first inequality follows from part (a), the second by domination, the third equation by rearranging terms, the fourth inequality from the facts that $z(u)+z(w) \leq$ $\bar{c}(u w)+1, \forall u w \in M$, and that $z(u)=-\lfloor\gamma \sqrt{n}\rfloor$ for $u \in U_{\text {free }}$ and $z(w)=0$ for $w \in W_{\text {free }}$, and the last inequality from the fact that $\bar{c}(u w) \leq 0$ for all $u w \in E$. Thus $\left|W_{\text {free }}\right|=\left|U_{\text {free }}\right| \leq 4 n /(\lfloor\gamma \sqrt{n}\rfloor) \leq 8 \sqrt{n} / \gamma$ for $n \geq 16$.

(d) To see that $\sum_{u} y(u)+\sum_{w} y(w)-c\left(M^{*}\right) \leq 4 n$, note that $\sum_{u} y^{\prime}(u)+\sum_{w} y^{\prime}(w)-$ $c\left(M^{*}\right) \leq 3 n$, for each edge $u w \in M, z(u)+z(w) \leq \bar{c}(u w)+1 \leq 1$, and, for each $v \in U_{\text {free }} \cup W_{\text {free }}, z(v) \leq 0$.

(e) Each iteration of the while-loop in phase 2 takes time $O(m)=O\left(n^{2}\right)$; see Section 2.1 of [GT] for details.

We now discuss the implementation of phase 1. We call an edge $u w z$-tight if $\bar{c}(u w)=$ $z(u)+z(w)$. Clearly, an edge $u w$ that is not $z$-tight can become $z$-tight only by a decrement of $z(u)$. Therefore, the search for $z$-tight edges in phase 1 can be done as follows: For each vertex $u \in U_{\text {free }}$ we maintain a pointer into $u$ 's adjacency list such that no edge to the "left" of the pointer is $z$-tight. If $u$ is selected in phase 1 the pointer is advanced until a $z$-tight edge, say $u w$, is found. This edge is added to $M$ and becomes non- $z$-tight by the increment of $z(w)$. If no $z$-tight edge is encountered, then $z(u)$ is decreased and $u$ 's 
pointer is reset to the first edge on $u$ 's adjacency list. In this way, each adjacency list is scanned at most $\gamma \sqrt{n}$ times for a total cost of $\gamma n^{2.5}$. Note, however, that only $O\left(\gamma n^{3 / 2}\right)$ $z$-tight edges are found in phase 1 according to Lemma 13(b). Therefore, we may hope to speed up the search for $z$-tight edges by the compression method. The details are as follows:

Let $d_{u w}=\bar{c}(u w)-z(u)-z(w)$. We maintain a matrix $D[u, w],(u, w) \in U \times W$, that approximates $d_{u w}$. More precisely, $D[u, w] \in[-q . .0] \cup\{-\infty\}$, where the value of $q=O(\log n)$ is to be fixed later, and $D[u, w]=0$ if and only if $u w \in E$ and $d_{u w}=0$. Let $b \geq\lceil\log (q+2)\rceil$, i.e., $b$ bits suffice to encode an entry of $D[u, w]$, and let $t \in \mathbb{N}$ be such that $t^{2} \cdot b \leq \log n$. The actual value of $t$ will be fixed later. We partition the matrix $D$ into $t \times t$ submatrices and store each submatrix in a single word of the RAC.

We now show how to maintain the invariant that $D[u, w]=0$ if and only if $u w \in E$ and $d_{u w}=0$, and how to search for $z$-tight edges. In order to maintain the invariant, we recompute for each $v \in U \cup W$. the row (if $v \in U$ ) or column (if $v \in W$ ) of $D$ corresponding to $v$ after every $q / 2$ changes of $z(v)$ according to the following rules:
If $v \in U, \quad$ then $D[v, w]=\left\{\begin{array}{l}d(v, w) \\ -\infty\end{array}\right.$
if $v w \in E$ and $d(v, w) \geq-q / 2$,
If $v \in W$, then $D[u, v]=\left\{\begin{array}{l}d(u, v) \\ -\infty\end{array}\right.$
if $v w \notin E$ or $d(v, w)<-q / 2$.
if $u v \in E$ and $\quad d(u, v) \geq-q / 2$.
if $u v \notin E$ or $d(u, v)<-q / 2$.

This takes time $O(n)$ for each recomputation and hence $O\left(\gamma n^{2.5} / q\right)$ time throughout phase 1.

Whenever $z(u), u \in U$, or $z(w), w \in W$, is changed and the above recomputation of (the compressed) $D$ is not done, then the appropriate row or column of $D$ has to be updated word by word using table lookup. We assume that $-\infty+1=-\infty$, and $-q-1=-q$. Given appropriate tables, updating one row or column takes time $O(n / t)$ for a total time of $O\left(n^{2.5} \cdot \gamma / t\right)$ throughout phase 1 .

Finally, we turn to the search for $z$-tight edges. Since the matrix $D$ reflects the zero values of $d$ correctly, we only need to search the (compressed) matrix $D$ for entries of value zero. Given appropriate tables, for each scan of an adjacency list this takes time $O(n / t+\sharp e)$, where $\ddagger$ e denotes the number of $z$-tight edges found. Therefore, the total time in Phase 1 for searching for $z$-tight edges is $O\left(n^{2.5} \gamma / t+\gamma n^{3 / 2}\right)$.

The various tables required for scanning/updating rows or columns of the compressed matrix $D$ word by word certainly can be computed in time $O\left(2^{2 t^{2} b}\right)$.

We summarize in:

LEMMA 14. The time complexity of a call of auction is

$$
T_{\text {auction }}=O\left(2^{2 t^{2} b}+\frac{n^{2.5} \gamma}{t}+\frac{n^{2.5} \gamma}{q}+\gamma n^{1.5}\right),
$$

where the constants $b, t$, and $q$ must satisfy $b \geq\lceil\log (q+2)\rceil$ and $t^{2} b \leq \log n$.

The choices $t=q=\left\lfloor(\log n / 2 \log \log n)^{1 / 2}\right\rfloor$ and $\gamma^{2}=t$ yield

$$
T_{\text {auction }}=O\left(n^{2.5}\left(\frac{\log \log n}{\log n}\right)^{1 / 4}\right) \text {. }
$$


We have thus proved:

THEOREM 8. An optimal assignment in a bipartite network $(U, W, E, c)$, where $|U|=$ $|W|=n$ and edge costs $c(u w) \in[0 . . C]$ for all $u w \in E$, can be computed in time $O\left(n^{2.5}(\log \log n / \log n)^{1 / 4} \log (n C)\right)$ by a conservative algorithm.

4. Conclusions and Open Problems. We showed that the parallelism at the wordlevel available in random access computers can be used to speed up many graph algorithms. In particular, we showed that a bipartite matching can be computed in time $O\left(n^{2.5} / \log n\right)$ and that the transitive closure of an acyclic digraph can be computed in time $O\left(n^{2}+n \cdot m_{\text {red }} / \log n\right)$.

For the transitive closure problem we also included some experimental evidence that the improvements are not only theoretical but also translate into smaller running times for practical problem sizes. We expect these improvements to become larger as the word size of machines increases.

Our methods easily extend to a few other algorithms for problems on graphs and networks: Prim's algorithm for finding minimum spanning trees [CLR, Section 24.2] can be accelerated to $O\left(n^{2} \log \max (2, C) / \log n\right)$, assuming that the matrix of edge costs is available in compressed form, using the method of Section 3.1. The scan-first search algorithm of [CKT] can be accelerated to $O\left(n^{2} / t+n t\right)$, assuming that the $t$-compressed adjacency matrix is available, hence, a sparse certificate for the $k$-connectivity of an undirected graph can be computed in time $O\left(k\left(n^{2} / t+n t\right)\right)$ under the same assumption; see [CKT] and Section 2.3 for details. Some interesting open questions are whether the fastest algorithms known for maximum-cardinality nonbipartite matching, finding 3-connected components, and constructing Gomory-Hu multiterminal flow trees, respectively, can be accelerated on the $\lambda$-RAC. We leave it as an open problem to characterize the class of graph problems and network problems for which bit-compression yields speed ups.

\section{References}

[ABMP] H. Alt, N. Blum, K. Mehlhorn, and M. Paul. Computing a maximum cardinality matching in a bipartite graph in time $O\left(n^{1.5} \sqrt{m / \log n}\right)$. Inform. Process. Lett., 37:237-240, 1991.

[AGOT] R. K. Ahuja, A. V. Goldberg, J. B. Orlin, and R. E. Tarjan. Finding minimum-cost flows by double scaling. Math. Programming, 53:243-266, 1992.

[AMO] R. K. Ahuja, R. L. Magnanti, and J. B. Orlin. Network flows. Handbook in Oper. Res. Management Sci., 1:211-360, 1991 .

[AV] D. Angluin and L. G. Valiant. Fast probabilistic algorithms for hamiltonian circuits and matching. J. Comput. Systems Sci., 18:155-193, 1979.

[B] D. P. Bertsekas. A new algorithm for the assignment problem. Math. Programming, 21:152-171, 1981.

[CHM] J. Cheriyan, T. Hagerup, and K. Mehlhorn. Can a maximum flow be computed in $o(n m)$ time. Proc. 17th ICALP Conference, pp. 235-248. Lecture Notes in Computer Science, vol. 443. SpringerVerlag, Berlin, 1990. The full version is available as Technical Report MPI-I-91-120, Max-PlanckInstitut fuir Informatik, Saarbricken.

[CKT] J, Cheriyan, M. Y. Kao, and R. Thurimella. Scan-first search and sparse certificates: an improved parallel algorithm for $k$-vertex connectivity. SIAM J. Comput., 22:157-174, 1993. 
[CLR] T. H. Cormen, C. E. Leiserson, and R. L. Rivest. Introduction to Algorithms. McGraw-Hill/The MIT Press, New York/Cambridge, MA, 1990.

[D1] E. W. Dijkstra. A note on two problems in connexion with graphs. Numer. Math., 1:269-271, 1959.

[D2] E. W. Dijkstra. Selected Writings in Computing: A Personal Perspective. Springer-Verlag, Berlin, 1982.

[FM] T. Feder and R. Motwani. Clique partitions, graph compression and speeding-up algorithms. Proc. 23rd ACM STOC; pp. 123-133, 1991.

[FW] M. L. Fredman and D. E. Willard. Blasting through the information theoretic barrier with fusion trees. Proc. 22nd ACM STOC, pp. 1-7, 1990.

[GK] A. Goralcikova and V. Koubek. A reduct and closure algorithm for graphs. Proc. Mathematical Foundations of Computer Science, pp. 301-307. Lecture Notes in Computer Science, vol. 74. Springer-Verlag, Berlin, 1979.

[GT] H. N. Gabow and R. E. Tarjan. Faster scaling algorithms for network problems. SIAM J. Comput., 18:1013-1036, 1989.

[HK] J. E. Hopcroft and R. M. Karp. An $n^{5 / 2}$ algorithm for maximum matchings in bipartite graphs. SIAM J. Comput., 2:225-231, 1973.

[KR] D. Kirkpatrick and S. Reisch. Upper Bounds for Sorting Integers on Random Access Machines. EATCS Monographs on Theoretical Computer Science, vol. 28, pp. 263-276. Springer-Verlag, Berlin, 1984.

[M] K. Mehlhorn. Data Structures and Efficient Algorithms, vol. I-III. Springer-Verlag, Berlin, 1984.

[MN] K. Mehlhorn and St. Näher. LEDA: A platform for combinatorial and geometric computing. Comm. ACM, 38(1):96-102, 1995.

[N] St. Näher. LEDA manual. Technical Report MPI-I-93-109, Max-Planck-Institut für Informatik, 1993.

[OA] J. B. Orlin and R. K. Ahuja. New scaling algorithms for the assignment and minimum mean cycle problems. Math. Programming, 54:41-56, 1992.

[Sh] M. Sharir. A strong-connectivity algorithm and its application in data flow analysis. Comput. Math. Appl., 7(1):67-72, 1981.

[Si] K. Simon. An improved algorithm for transitive closure on acyclic digraphs. Proc. 13th ICALP Conference, pp. 376-386. Lecture Notes in Computer Science, vol. 226. Springer-Verlag, Berlin, 1986.

[T] R. E. Tarjan. Depth-first search and linear graph algorithms. SIAM J. Comput., 1:146-160, 1972. 